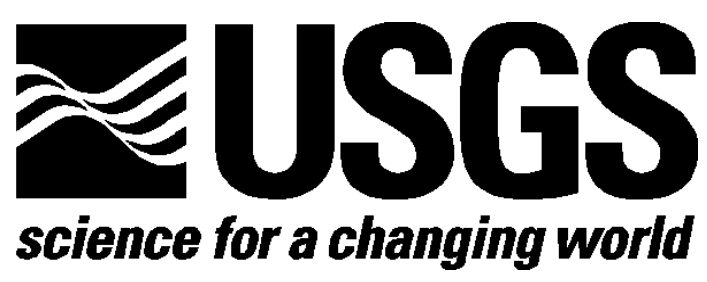

\title{
McVCO Handbook 1999
}

by P. J. McChesney ${ }^{1}$

Open File Report 99-361

1999

This report is preliminary and has not been reviewed for conformity with U. S. Geological Survey editorial standards. Any use of trade, firm, or product names is for descriptive purposes only and does not imply endorsement by the U. S. Government. Although this device and its contained program have been used by the U.S. Geological Survey, no warranty, expressed or implied is made by the USGS as to the accuracy and functioning of the device, its program and related program material, nor shall the fact of distribution constitute any such warranty, and no responsibility is assumed by the USGS in connection therewith.

Department of the Interior

U. S. Geological Survey

${ }^{1}$ University of Washington Geophysics Program

USGS Cascades Volcano Observatory

5400 MacArthur Blvd.

Vancouver WA 98661
University of Washington

Geophysics Program 


\section{U.S. Department of the Interior \\ Bruce Babbit, Secretary}

\section{U.S. Geological Survey}

Charles Groat, Director

This report is only available in digital form on the World Wide Web. URL: http://wrgis.wr.usgs.gov/open-file/of99-361 


\section{Contents}

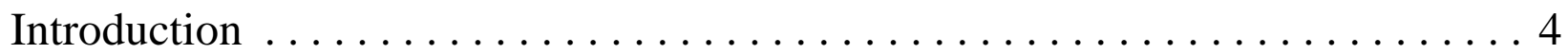

Part I: McVCO Operation $\ldots \ldots \ldots \ldots \ldots \ldots \ldots \ldots \ldots \ldots \ldots \ldots \ldots \ldots \ldots \ldots \ldots \ldots$

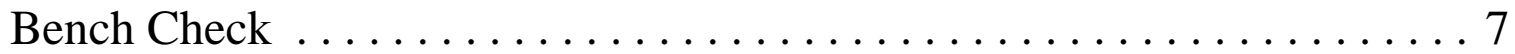

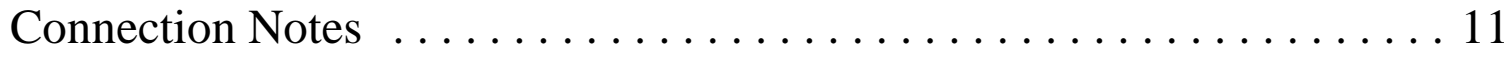

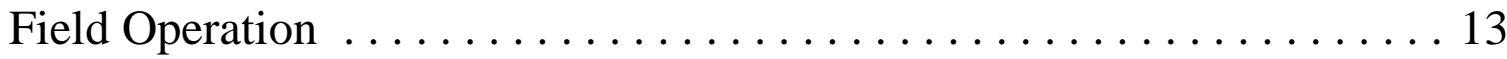

Setup Procedure ............................ 15

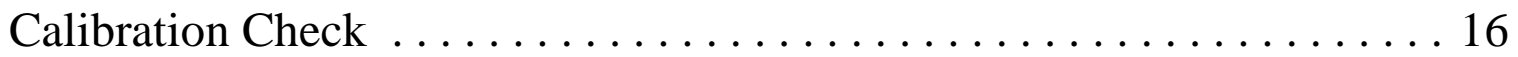

Figure 2A. Part locations and DIP switch $\ldots \ldots \ldots \ldots \ldots \ldots \ldots \ldots$

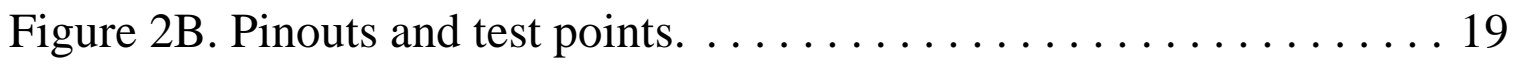

Part II: Technical Reference . . . . . . . . . . . . . . . . . . . . . 20

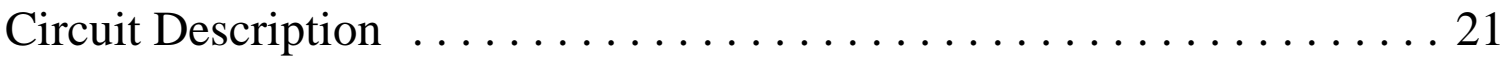

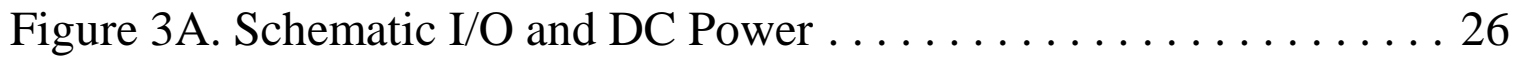

Figure 3B. Schematic Seismic-signal Amplifier . . . . . . . . . . 27

Figure 3C. Schematic ADC, Microcontroller and Sinewave Generator . . 28

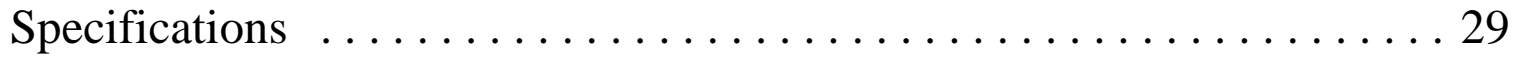

Part III: Application Notes . . . . . . . . . . . . . . . . . . . . 32

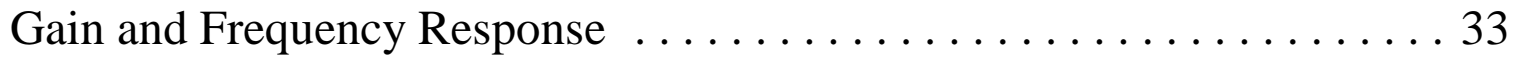

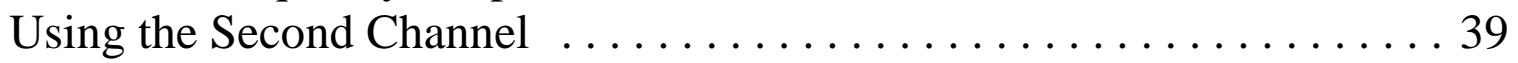

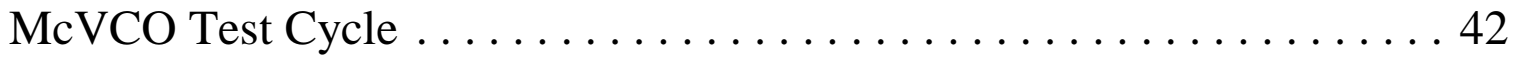

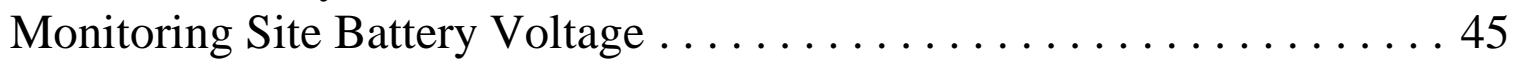

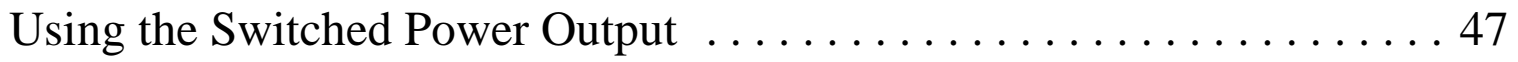

Three Component Connection $\ldots \ldots \ldots \ldots \ldots \ldots \ldots \ldots$ 


\section{Introduction}

$\mathrm{McVCO}$ is a microcontroller-based frequency generator that replaces the voltage controlled oscillator (VCO) used in the analog telemetry of seismic data. It accepts low-level signals from a seismometer and produces a frequency modulated subcarrier suitable for radio or telephone links to a data collection site. The gain and subcarrier frequency are switch selectable. Two channel operation for low and high gain observation from a single instrument is an option. Figure 1 shows the block diagram of the instrument.

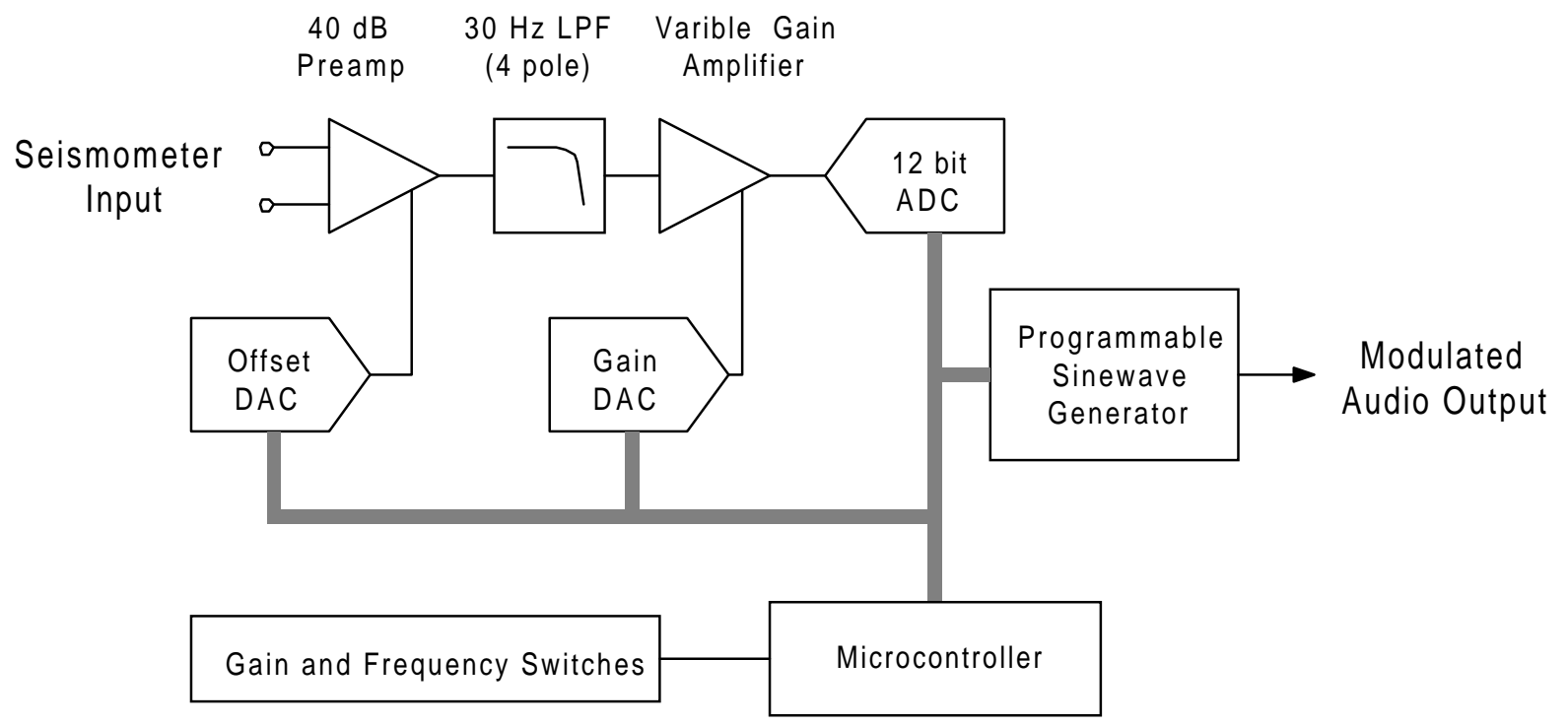

Figure 1. Microcontroller based VCO (McVCO) block diagram.

Unlike a VCO made with analog circuits, McVCO uses analog to digital conversion (ADC) to sample the seismic-signal amplifier output. The value of each sample determines the code sent to the programmable sinewave generator. The generator synthesizes the required sinewave frequency so that it is proportional to the sample. The sample and synthesize cycle repeats several hundred times each second to produce an audio signal that is frequency modulated by the seismic signal. The advantage of McVCO's method of producing a modulated signal is its freedom from the component aging and temperature effects that reduce the stability of analog VCOs.

Besides calculating modulation, the microcontroller runs an offset reduction scheme which further increases McVCO stability. Offsets created by the analog components of the seismicsignal amplifier are subtracted by the offset digital to analog converter (DAC) at the input 
instrumentation amplifier. Offset removal assists operation at the chosen center frequency. Another DAC provides microcontroller gain control. This allows the instrument to adapt to various modulation sensitivity standards by means of gain tables.

$\mathrm{McVCO}$ was designed for the purpose of improving the analog telemetry of signals within the Pacific Northwest Seismograph Network (PNSN). Its development received support from the University of Washington Geophysics Program, and both the Volcano Hazards and Earthquake Hazards programs of the United States Geological Survey (USGS). Several hundred instruments have been built and deployed. In addition to use in the PNSN, McVCO is used by the Alaska Volcano Observatory in monitoring Aleutian volcanos and by the USGS Volcano Disaster Assistance Program in responding to volcanic crises overseas.

Part I of this handbook covers operation of McVCO. It aims at giving the user enough information to connect a unit, check its operation, and install it in the field. McVCO has some characteristics that are unlike the analog VCO. Consequently, the Bench Check section is strongly recommended for new users because it demonstrates McVCO's unique control and operating qualities. Figure $\mathbf{2 A}$ and $\mathbf{2 B}$ are summaries of the information required to operate the instrument successfully.

Part II of the handbook is a technical reference for those who require a closer look at how $\mathrm{McVCO}$ works. An experienced technician will find enough detail to diagnose and repair most faults.

Part III covers a collection of topics that need explicit treatment or that spring from deployment of the instrument. It is expected that these application notes will expand and McVCO users are urged to communicate their questions and experiences.

It is the intention of this handbook to provide the information required to understand and operate $\mathrm{McVCO}$. Those wishing to construct their own instruments from the information presented here are welcome to do so. However, the author expressly disclaims the implied warranties of merchantability and of the fitness for any particular purpose. It is the responsibility of the instrument maker to test and verify the operation of an instrument. The specifications given in this handbook are for guidance only and not a warranty of performance.

The microcontroller code required to build McVCO is not included in this handbook. Those wishing to buy programmed microcontrollers, or discuss McVCO may contact:

\author{
P. J. McChesney \\ Stone Code \\ 305 E $24^{\text {th }}$ Street \\ Vancouver, WA 98663 \\ USA
}




\section{Part I: McVCO Operation}




\section{Bench Check}

Checking McVCO on the bench requires the following:
A) 12 VDC power supply or battery.
B) Audio-range frequency counter or frequency-reading digital multi-meter (DMM) with $1 \mathrm{~Hz}$ resolution.
C) $1.5 \mathrm{VDC}$ battery, $100 \Omega$ and $100 \mathrm{~K} \Omega$ resistors. Use $1 \%$ resistors for the calibration check.

\section{DO NOT APPLY POWER UNTIL TOLD TO DO SO.}

Examine Figure $2 A$ at the end of Part I. The top section has a graphic that shows the location of major McVCO components. In particular note the location of the DIP switch in the upper right corner of the board and the reset pins on the lower edge of the board toward the right.

\section{1) Setting the DIP Switch}

The DIP switch controls gain and subcarrier frequency. The top switch is switch 1 and the bottom is switch 9. Switches 1, 2 and 3 control the gain of the variable-gain channel. Switches 4, 5 and 6 control the variable-gain channel subcarrier frequency, f1. When the board is equipped for two channel operation, switches 7,8 and 9 control the fixed low-gain subcarrier frequency, f2. The McVCO printed circuit board is marked to show the switch functions.

At the bottom of Figure $2 A$ are the switch patterns for the various gain and frequency selections. A switch set to 0 means the switch is closed and the switch lever is thrown toward the edge of the board. A switch set to 1 is open and its lever is toward the center of the board.

Set the switches so that the gain is $60 \mathrm{~dB}, \mathrm{f} 1=680 \mathrm{~Hz}, \mathrm{f} 2=3060 \mathrm{~Hz}$

$\begin{array}{llllllllll}\text { switch } & 1 & 2 & 3 & 4 & 5 & 6 & 7 & 8 & 9 \\ & 0 & 1 & 1 & 0 & 0 & 0 & 1 & 1 & 1\end{array}$

Power to the board is connected with the positive side of the supply or battery going to B+ and the negative to GND. B+ and GND are found in the lower left corner of the board and are marked solder points. If the board is packaged, consult the wiring and connector diagrams for the correct hookup.

Connect a frequency counter between TP5 and GND and TURN ON THE POWER. The counter shows $680 \mathrm{~Hz}$ for 10 seconds while the instrument autozeros. After 10 seconds the unit begins a test routine that lasts 42 seconds and the count will shift above and below $680 \mathrm{~Hz}$. After the test routine the output will settle to $680 \mathrm{~Hz} \pm 2 \mathrm{~Hz}$. Early versions of the program do not run the 42second test routine and some later versions have longer test periods. 


\section{2) $\underline{\text { Reset }}$}

Change the DIP switch to $\mathrm{Sw} 4=0, \mathrm{Sw} 5=0, \mathrm{Sw} 6=1$. Notice that there is no change in frequency. Nothing happens when DIP switches are changed until the unit is RESET. Find the reset pins on the lower edge of the board on the right. Touch both pins at the same time with a conductive object such as a probe tip or screwdriver blade. The unit resets when the short between the pins is removed.

RESET the unit. The frequency now changes to $1020 \mathrm{~Hz}$ and after the $10 \mathrm{~s}$ autozero and the $42 \mathrm{~s}$ test routine the count will settle to within $2 \mathrm{~Hz}$ of $1020 \mathrm{~Hz}$.

\section{3) Changing Frequency}

The switch patterns for the other $\mathrm{f1}$ frequencies are given in the middle table on Figure $2 \mathrm{~A}$. Work through them. Unless $3060 \mathrm{~Hz}$ is selected, the frequency will change each time the unit is reset.

When $3060 \mathrm{~Hz}$ is selected for f1 the unit will go into a special shutdown mode, and the counter will read $0 \mathrm{~Hz}$. Look at switches 7, 8, and 9 and compare them with 4, 5, and 6. They are the same and the switch is set so $\mathrm{f} 1=\mathrm{f} 2$. Whenever Sw4 $=\mathrm{Sw} 7$ and Sw5 = Sw8 and Sw6 = Sw9, the unit will go into a shutdown mode used for some adjustments (see Setup Procedure). This happens even if $\mathrm{f} 2$ is not used. If the unit produces no output, always check to see if the DIP switch is set for $\mathrm{f} 1=\mathrm{f} 2$ and in shutdown mode.

Change any $\mathrm{f} 2$ switch $(7,8,9)$. Reset and observe that the unit is no longer shut down and the frequency counter reads $3060 \mathrm{~Hz} \pm 2 \mathrm{~Hz}$.

The second channel is active if the $\mathrm{f} 2$ generator chip, U16, is installed, and pin 16 of the microcontroller, U9, is jumpered to +5VDC (see Figure $2 A$ for location). If it is, work through the $\mathrm{f} 2$ switch pattern after moving the counter to TP6. The $\mathrm{f} 2$ switches work the same way that the f1 switches do.

\section{4) Changing Gain}

Prepare a signal source of approximately $1.5 \mathrm{mV}$ by connecting the $100 \mathrm{~K} \Omega$ and $100 \Omega$ resistors in series across the $1.5 \mathrm{~V}$ battery. Connect the positive side of the battery to one side of the 100 $\mathrm{K} \Omega$ resistor. Connect the negative side of the battery to one side of the $100 \Omega$ resistor. Join the free ends of both resistors. The $1.5 \mathrm{mV}$ signal source is the voltage across the $100 \Omega$ resistor. 
Set the dip switches to $\mathrm{G}=60 \mathrm{~dB}, \mathrm{f} 1=680 \mathrm{~Hz}, \mathrm{f} 2=3060 \mathrm{~Hz}$

$\begin{array}{llllllllll}\text { switch } & 1 & 2 & 3 & 4 & 5 & 6 & 7 & 8 & 9 \\ & 0 & 1 & 1 & 0 & 0 & 0 & 1 & 1 & 1\end{array}$

Connect the frequency counter to TP5 and RESET the unit. Wait about a minute for the autozero and test cycle to finish, then record the f1 frequency. It will be $680 \mathrm{~Hz} \pm 2 \mathrm{~Hz}$.

Connect the $1.5 \mathrm{mV}$ source with the negative side of the battery to I- and the connection between the resistors to I+.

The frequency will increase to a value between 720 and $760 \mathrm{~Hz}$. The increase depends on the exact battery voltage and the calibration used for your system. Note the change in frequency.

It is necessary to disconnect the $1.5 \mathrm{mV}$ source each time the unit is reset because each reset causes an autozero. If the $1.5 \mathrm{mV}$ source is connected during the autozero, the amplifier will try to null out the $1.5 \mathrm{mV}$ input.

Disconnect the $1.5 \mathrm{mV}$ source and set the gain for $54 \mathrm{~dB}$ Sw1 $=0, \mathrm{Sw} 2=1, \mathrm{Sw} 3=0$.

RESET the unit. Wait about a minute and reconnect the $1.5 \mathrm{mV}$ source exactly as it was previously. Observe that the change in frequency is half of what it was. As the gain is further lowered to $48 \mathrm{~dB}$ the change in frequency will halve again, showing that each $6 \mathrm{~dB}$ decrease in gain results in half the frequency change.

Disconnect the $1.5 \mathrm{mV}$ source and set the gain for $84 \mathrm{~dB}, \mathrm{Sw} 1=1, \mathrm{Sw} 2=1, \mathrm{Sw} 3=1$. RESET the unit and wait a minute before reconnecting the $1.5 \mathrm{mV}$ source. The frequency will change to around $800 \mathrm{~Hz}$. No matter how badly over driven, McVCO will not shift the subcarrier frequency further than $125 \mathrm{~Hz}$ from the center frequency.

\section{5) Autozero and Offset Compensation}

To observe the action of the autozero circuit, set the gain to $60 \mathrm{~dB}$ Sw1 $=0, \mathrm{Sw} 2=1, \mathrm{Sw} 3=1$ and with the $1.5 \mathrm{mV}$ source connected, RESET the unit. The frequency of f1 at TP5 goes to the channel center frequency, $680 \mathrm{~Hz} \pm 1 \mathrm{~Hz}$ for $10 \mathrm{~s}$ while the autozero takes place. At the end of the autozero and the test routine, the frequency will settle at some value greater than the correct center frequency. The $1.5 \mathrm{mV}$ input is too big for the offset correction circuit to zero.

Disconnect the $1.5 \mathrm{mV}$ source. The frequency will decrease to some value below the center frequency. This is the offset due to the bad null during autozero. Sometimes bad nulls occur in the field, see the discussion in the Field Operation section in Part I. 
The bad null can be removed in two ways. First, the unit runs an offset nulling scheme in the background during normal operation. The action of this scheme is very gradual since it is primarily intended to cancel offsets due to temperature changes and component aging. Details of the offset compensation scheme are given in the discussion of frequency response in Part III, McVCO Gain and Frequency Response.

To observe the background offset compensation, create a bad null by resetting the unit while it is connected to the $1.5 \mathrm{mV}$ source. Remove the $1.5 \mathrm{mV}$ input and wait. It takes a little more than $18 \mathrm{hrs}$ to return to center frequency from an extreme offset. Leave the unit overnight or go to lunch. See how far the frequency has shifted toward the center frequency when you return.

The second and fastest way to get rid of a bad null is to RESET the unit without the $1.5 \mathrm{mV}$ connected and let it re-zero itself. Do not try to null the unit with P1, the null pot, without going through the Setup Procedure that follows in Part I.

You have now seen the basic operation of McVCO. As shown in Figure 2A, the DIP switch selects gain and frequency. Reset causes the switches to be read and starts an autozero and test cycle which lasts about a minute before McVCO begins normal operation. During operation, offset is removed by a long-term compensation scheme. The test cycles differ among the versions of McVCO. See McVCO Test Cycle in Part III for a description. 


\section{Connection Notes}

McVCO is not supplied in a standard package and most users supply the enclosure, connectors, and weatherproofing appropriate to their circumstances.

The design of McVCO makes it relatively insensitive to electrical interference and the primary consideration for packaging is protection from damp and mechanical stress. The degree of protection required is left to the common sense of the user. What follows are some suggestions about electrical connection and descriptions of some hookup options. See Figure $2 A$ for hookup locations.

\section{Seismometer Hookup}

McVCO expects to see a two-wire input with both sides isolated from earth ground. The two wires should be run in a shielded cable. The wire connected to the positive side of the seismometer coil connects to I+ on McVCO. The wire connected to the negative side of the coil connects to I-. If needed, a solder point connected to McVCO GND is provided between I+ and I- for the seismometer cable shield.

Do not allow the seismometer shield to become a current carrier. Make sure that it is tied to ground at only one place. If the shield is tied to ground at a connector shell, do not ground it at I+ and $\mathrm{I}^{-}$. The shield should extend close to I+ and I- even if it is not tied to ground there.

\section{$\underline{\text { Power Connections }}$}

\section{Power and Output}

The recommended connections for power are at the B+ and GND solder points with the positive power lead connected to $\mathrm{B}+$. The $\mathrm{B}+$ solder point is reverse-polarity protected by a series diode. For boards marked MCFM05, B+ found on the pins of J1 and J2 is on the opposite side of the protection diode from the $\mathrm{B}+$ solder point. More recent boards, marked McVCO-01, have the $\mathrm{B}+$ solder point and the $\mathrm{B}+$ on $\mathrm{J} 1$ and $\mathrm{J} 2$ directly connected.

$\mathrm{SW}+$ is a switched power output. It provides the input $\mathrm{B}+$ voltage minus about $0.5 \mathrm{VDC}$ at 200 $\mathrm{mA}$. SW+ switches off when the input power source falls below about 10.5 VDC and does not switch on until the power recovers to about 11.5 VDC.

$\mathrm{SW}+$ must be used with caution. If there is enough resistance in the connections between $\mathrm{McVCO}$ and the power source, the circuit will oscillate. This happens when the voltage drop across the resistive connection to the power source is greater than the 1 volt difference between the turn off and turn on levels. For example a transmitter powered from SW+ draws $80 \mathrm{~mA}$. This current plus McVCO's current combine for a total current of $100 \mathrm{~mA}$. The resistance between McVCO and the power source must be kept less than $10 \Omega$, ( $\mathrm{R}=1 \mathrm{VDC} / 0.1 \mathrm{~A})$. For 
an adequate safety margin the connection resistance should be less than $5 \Omega$ with a $100 \mathrm{~mA}$ total load. The wiring at most installations is much less than this but corrosion can increase the total resistance and cause oscillation.

Do not use SW+ when air cells or other resistive supplies are used as the power source. The circuit will oscillate as the internal voltage drop of the supply trips the ON and OFF switching levels of the circuit. See the Part III, "Using the Switched Power Output, SW+" for a fuller discussion.

\section{$\underline{\text { Subcarrier Outputs }}$}

The audio subcarrier is provided on Car+ and Car-. Both connections float with respect to ground. If a ground referenced output is wanted, Car- may be connected to ground either off the board or at the solder pads provided near the lower left corner of the output transformer, T1.

Car+ is capacitively coupled to the output transformer to provide DC isolation. Like any capacitively coupled output, this can lead to unwanted clamping with some loads. If this occurs, the coupling capacitor, C35, can be jumpered. Another remedy is to place a discharge resistor across Car+ and Car- ${ }^{-}$. Finally using an input coupling transformer at the load prevents unwanted clamping of McVCO's output.

\section{$\underline{\mathrm{J} 1 \text { and } \mathrm{J} 2}$}

Optional input and output connections are provided by $\mathrm{J} 1$ and $\mathrm{J} 2$. These are 8-pin modular jacks. See Figure $2 B$ for the pin outs. $\mathrm{J} 1$ is an output connection for power and the audio subcarrier. When a transmitter is packaged in the same enclosure with McVCO, modular telephone cable makes a convenient connection. J2 provides an input connection for audio subcarriers generated by other equipment. McVCO's output stage is a summing amplifier and signals appearing at $f_{\text {in }}$ on $\mathrm{J} 2$ are summed with the signal generated by McVCO.

By using J1 with J2, a three component site can be easily wired. Three McVCOs are chained with the $\mathrm{J} 1$ output of the first board connected to the J2 input of the second. The second board's $\mathrm{J} 1$ is connected to $\mathrm{J} 2$ of the third. The third board's output then has the subcarriers for all three components. See Part III, Three Component Connection for details.

\section{$\underline{S \text { and T Header }}$}

For installations that calibrate the seismometer response with an attenuator at the seismic-signal amplifier input, a socket is provided for $\mathrm{S}$ and $\mathrm{T}$ resistors, see Figure $2 \mathrm{~B}$. To preserve common mode noise rejection, the $\mathrm{T}$ resistance is split into two equal parts and becomes resistors $\mathrm{T} 1$ and $\mathrm{T} 2$. The $\mathrm{T}$ resistors are in series with the I input connections and the input amplifier. If $\mathrm{T}$ resistors are not used or are located off the board, then each T resistor position of the socket must be bridged by jumper wire or the input will be disconnected. Care and good soldering practices should be used when wiring the header. An open or intermittent connection will ruin the whole installation. 


\section{Field Operation}

All that is required to put $\mathrm{McVCO}$ into operation is to make the seismometer, power, and output connections and to set the DIP switch for gain and frequency. Connections are covered in the section entitled Connection Notes and setting the DIP switch is covered in the Bench Check section. Figure $2 A$ and $2 B$ summarize this information.

This section covers two topics: finding the correct gain setting, and what to do if McVCO does not immediately run within a few $\mathrm{Hz}$ of the nominal center frequency.

\section{$\underline{\text { Gain }}$}

If $\mathrm{McVCO}$ is replacing an instrument at an existing site then all that is required is to translate the old gain setting to McVCO's setting. A complication in doing this is that many older instruments use attenuators whose setting must be subtracted from the unattenuated gain of the instrument to find the operating gain. For example, the USGS J502 has an unattenuated gain of $90 \mathrm{~dB}$. If the attenuator is set to $18 \mathrm{~dB}$ then the gain is $72 \mathrm{~dB}(90 \mathrm{~dB}-18 \mathrm{~dB})$. The McVCO gain setting for the same response is $72 \mathrm{~dB}$.

If you are unable to find the old gain setting or $\mathrm{McVCO}$ is being installed at a new site, then the gain setting must be found from observing the seismic background. TP2 is provided for looking at the seismic signal with an oscilloscope. The signal at TP2 is similar to the signal at the discriminator output. It has the opposite phase and approximately the same amplitude. The amplitude at the discriminator will depend on both the modulation and demodulation sensitivities.

Connect an oscilloscope to TP2 and find the gain setting which gives an acceptable background signal level. A good gain to start at is $66 \mathrm{~dB}$ and many sites are set up for a peak to peak background signal of 50 to $100 \mathrm{mV}$. Remember to RESET after each change in the DIP switches and wait a minute for autozero and test routines to finish.

\section{Frequency}

Very rarely, the autozero after reset will fail to cause f1 to run within $2 \mathrm{~Hz}$ of the nominal center frequency. This is usually because a strong signal occurred at the end of the autozero period, or the background seismic signal has strong long period components. The remedy is simple. Reset the unit and avoid moving during the 10-second autozero period that follows. Even with long period background present, a couple of tries should give a good center frequency.

If $\mathrm{McVCO}$ is packaged with a transmitter and is operating at high gain, the center frequency can shift when covers are closed. Radio carrier leakage into McVCO circuits usually appears as a DC component in the seismic signal amplifier. Try closing the covers and interrupting power to force a reset and autozero with the radio and $\mathrm{McVCO}$ in their operating conditions.

If the transmitter is switched through SW+ the unit will autozero before the transmitter is on. 
This may cause a temporary offset but the long-term offset compensation scheme will pull the average f1 frequency to the nominal center channel frequency. So do not worry, McVCO self adjusts if there is no time to fiddle around with it.

There are two situations in which a functional McVCO will fail to autozero. At some sites where the antenna is not grounded at the mast, the seismometer input cable can pickup enough RF energy to upset the seismic-signal amplifier. If the McVCO zeros when the transmitter is off, bad antenna grounding is the problem. Fix it.

The other cause of autozero problems is seismometer corrosion. In particular, corrosion where the coil leads pass through the case inside the L4 seismometer can create an electrochemical cell strong enough to unbalance the seismic-signal amplifier. If the McVCO autozeros with the seismometer disconnected, suspect a corrosion cell. The presence of the cell is shown by a DC voltage between either side of the coil and the seismometer body. Cell outputs are intermittent and temperature sensitive.

McVCO has a directly coupled seismic-signal amplifier. DC voltage applied to the amplifier inputs appears as offset in the amplifier output. Offsets are corrected by the offset correction feedback loop but the maximum DC input voltage that the circuit can null is $\pm 0.5 \mathrm{mV}$.

Unfortunately, corrosion cells can produce tens of millivolts at the seismic-signal amplifier input. This is enough to overdrive the input stage of most seismic-signal amplifiers even when later stages are capacitively coupled.

If the cell is inactive, corrosion can be detected with an ohmmeter. The manufacturer, Mark Products, specifies leakage between the coil and the L4 case as greater than $100 \mathrm{M} \Omega$ at 500 volts. Corrosion cells produce resistance readings less than $10^{6} \Omega$ with an ordinary ohmmeter. Disconnecting the cable shield from the seismometer body or McVCO ground may disconnect the cell if corrosion is only on one side of the coil. However, once corrosion starts on one side of the coil, it usually develops on the other side and completes the circuit to the amplifier input. Corrosion cell problems may be intermittent but they do not fix themselves. Usually corrosion grows and the problem gets worse. Seismometers with signs of corrosion should be replaced.

In a difficult situation, the offset adjustment range of the instrument can be increased by a factor of 4. To increase the range, cut the lead for R20 at the upper board edge. This will raise the reference voltage for the offset null circuit from 50 to 200 millivolts. The autozero will now tend to overshoot when the instrument is reset and noise from the background null may increase. However, the greater offset null range may succeed as a temporary measure in repairing a site that needs a better antenna ground or a seismometer without internal corrosion.

The second channel subcarrier, $\mathrm{f} 2$, is not part of the autozero or offset compensation schemes. When it is used, it is a low gain channel and is consequently stable. The shift2 adjustment, P4, can be used to adjust $\mathrm{f} 2$ to the channel center frequency, if necessary. 


\section{Setup Procedure}

Correct operation of McVCO depends on setting up the null and level shift circuits in the seismic signal amplifier. These adjustments establish the operating point around which the autozero and offset compensation schemes work. An incorrect setup decreases the ability of these routines to maintain operation in the center of the channel.

The setup should be done at a temperature in the middle of the range in which the unit is expected to operate. Room temperature is fine for most situations. Checking the setup at the bench before taking a unit into the field only requires a few minutes and is recommended. A $3 \frac{1 / 2}{2}$ digit, digital volt meter (DVM) is required.

See Figure $2 A$ for adjustment locations.

1) Place the unit in shutdown mode by setting the DIP switches so that $\mathrm{f} 1=\mathrm{f} 2$ and set the gain for $66 \mathrm{~dB}$.

2) Apply power or reset the unit

3) Connect a DVM between GND and TP1 and adjust P1, Null, for 0 VDC $\pm 10 \mathrm{mV}$.

4) Connect a DVM between GND and TP3 and adjust P3, Shift1, for $2.50 \mathrm{VDC} \pm 10 \mathrm{mV}$.

5) Connect a DVM between GND and TP4 and adjust P4, Shift2, for $2.50 \mathrm{VDC} \pm 10 \mathrm{mV}$.

This completes the setup. 


\section{Calibration Check}

The calibration can be checked and adjusted with simple equipment. Required are a 31/2 digit, digital multi-meter (DMM); an audio-range frequency counter; and a 1.5mVDC source like that used in the Bench Check section of this manual. Do not adjust the calibration without completing the Setup Procedure above.

1) To perform the calibration it is necessary to know the modulator deviation sensitivity for your seismic network in $\mathrm{Hz} / \mathrm{V}$. Find this out before going further. The J502 and J512 produced by the USGS use $115 \mathrm{~Hz} / 4.05 \mathrm{~V}$ and McVCOs use this deviation sensitivity for USGS installations. Another common deviation sensitivity is $125 \mathrm{~Hz} / 3.00 \mathrm{~V}$.

2) Prepare a $1.5 \mathrm{mVDC}$ source by connecting $100 \Omega$ and $100 \mathrm{~K} \Omega 1 \%$ resistors across a $1.5 \mathrm{VDC}$ battery. Connect one end of the $100 \Omega$ resistor to the negative side of the battery. Connect one end of the $100 \mathrm{~K} \Omega$ resistor to the positive side of the battery. Connect the free ends of the two resistors together. The $1.5 \mathrm{mVDC}$ source is across the $100 \Omega$ resistor.

3) Measure the battery voltage of the $1.5 \mathrm{mV}$ source. The resistors divide the battery voltage by 1000. When the unit's gain is set for $60 \mathrm{~dB}(1000)$, the change in frequency $(\Delta \mathrm{f})$ is (battery voltage) $\mathrm{x}$ (deviation sensitivity). Do this calculation.

4) If the unit uses a header with $T$ resistors, replace this header with one that has shorting jumpers in the $\mathrm{t} 1$ and $\mathrm{t} 2$ header positions. Gain calibration is referenced to the seismic-signal amplifier input and does not include S and T header attenuation. See "McVCO Gain and Frequency Response" in Part III for a fuller discussion.

5) Set the unit for gain $=60 \mathrm{~dB}$. Any value of $\mathrm{f} 1$ center frequency may be set since the calibration is valid for any selected frequency. If a frequency reading DMM is used, select a low value of $\mathrm{f} 1$ to maximize the resolution of your $\mathrm{f} 1$ measurement. Do not attempt to adjust the calibration without a frequency measurement with $1 \mathrm{~Hz}$ resolution.

6) Connect test leads to the I+ and I- seismometer inputs and short their free ends together. Apply power to the unit or reset it. Wait a minute for the autozero and test cycle to finish and record the operating center frequency (fc) measured at TP5. This should be within $2 \mathrm{~Hz}$ of the selected $\mathrm{f} 1$.

7) Calculate the positive and negative deviation of $\mathrm{f} 1$ from $\Delta \mathrm{f}$ in step 3 and fc in step 6.

$$
\begin{aligned}
& + \text { Dev }=f c+\Delta f \\
& - \text { Dev }=f c-\Delta f
\end{aligned}
$$


8) Apply the $1.5 \mathrm{mVDC}$ source to the seismometer input with the test leads. Connecting the negative side of the battery to I- with I+ wired to the connection between the two resistors, gives a positive deviation. Reversing the connection gives a negative deviation. Compare calculated to measured deviations.

9) Calculated and measured deviations should be within $1 \mathrm{~Hz}$ of each other. Adjust the cal. pot, $\mathrm{P} 2$, if required and repeat the check for the other input polarity. Adjust until both deviations are within $1 \mathrm{~Hz}$ of their calculated values. Start again at step 6 if adjustment is unsuccessful. When repeating this procedure, performing the reset in step 6 is not necessary, but checking the center frequency with the input shorted is important.

This method gives calibration accuracy to within $3 \%$ across all gain ranges and is independent of the $\mathrm{f} 1$ frequency. Better adjustment can be obtained with a $60 \mathrm{~dB}$ attenuated $2.5 \mathrm{~V}$ reference instead of the battery and resistors. No calibration adjustment of the user defined second channel is provided. 
Part 1: McVCO Operation

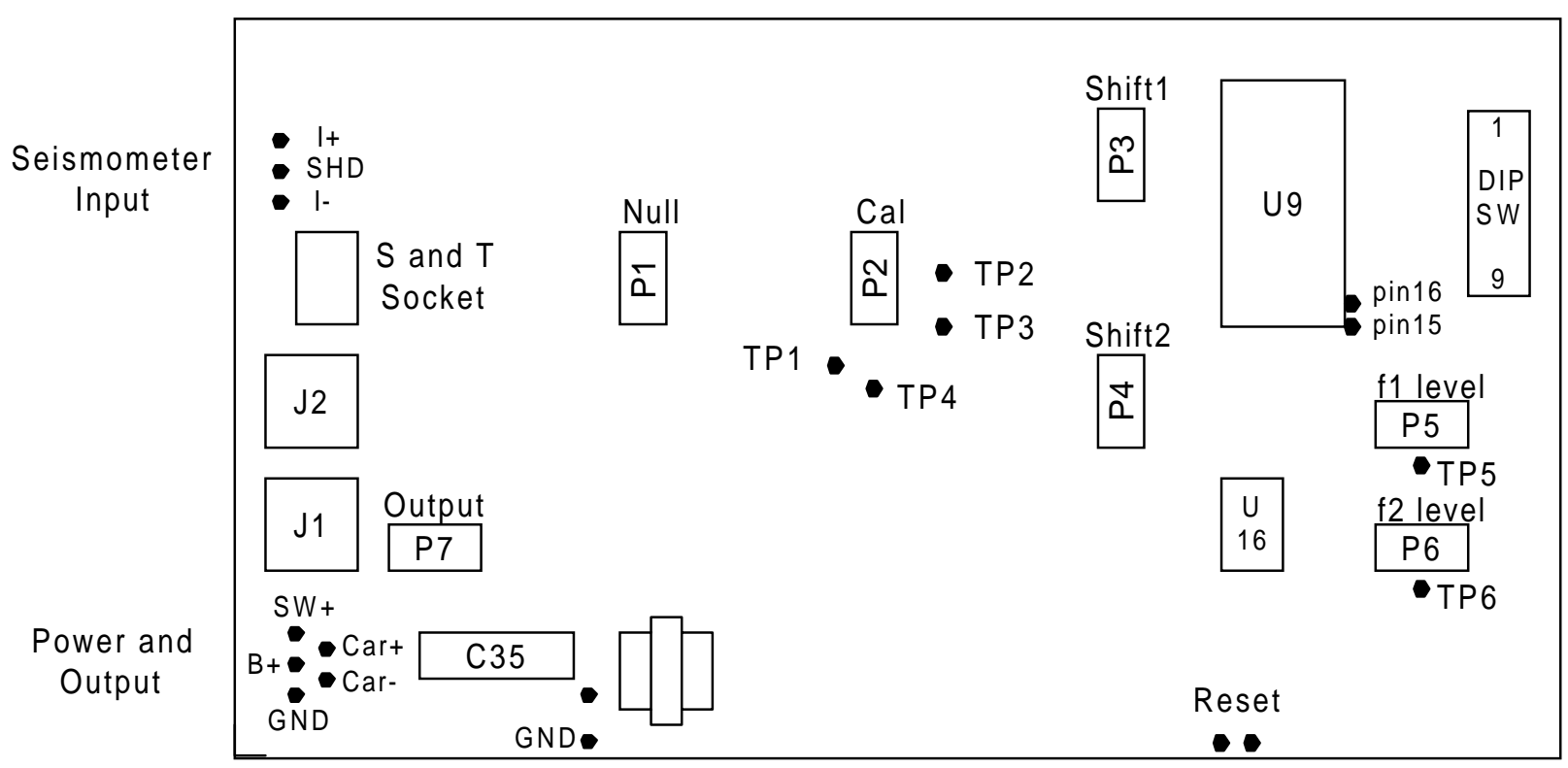

I/O Labels $\quad B+$ is the positive power supply connection, nominally +12 volts. GND is the negative power supply connection and is 0 volts. $\mathrm{SW}+$ is the switched unregulated +12 volt power supply. $\mathrm{Car}+$ and Car- are the audio subcarrier outputs.

$\mathrm{I}+$ is the connection for the positive side of the seismometer coil. $\mathrm{I}$ - is the connection for the negative side of the seismometer coil. SHD is the connection for the seismometer cable shield.

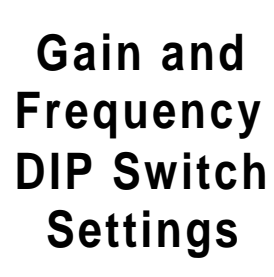

\begin{tabular}{|c|c|}
\hline $\begin{array}{c}\text { GAIN } \\
(\mathrm{dB})\end{array}$ & $\begin{array}{c}\text { SW } \\
123\end{array}$ \\
\hline 42 & 000 \\
\hline 48 & 001 \\
\hline 54 & 010 \\
\hline 60 & 011 \\
\hline 66 & 100 \\
\hline 72 & 101 \\
\hline 78 & 110 \\
\hline 84 & 111 \\
\hline
\end{tabular}

\begin{tabular}{|c|ccc|}
\hline$f 1$ & \multicolumn{2}{|c|}{$S \mathrm{~W}$} \\
$(\mathrm{~Hz})$ & 4 & 5 & 6 \\
\hline 680 & 0 & 0 & 0 \\
1020 & 0 & 0 & 1 \\
1360 & 0 & 1 & 0 \\
1700 & 0 & 1 & 1 \\
2040 & 1 & 0 & 0 \\
2380 & 1 & 0 & 1 \\
2720 & 1 & 1 & 0 \\
3060 & 1 & 1 & 1 \\
\hline
\end{tabular}

\begin{tabular}{|r|lll|}
\hline$f 2$ & \multicolumn{2}{|c|}{ SW } \\
$(\mathrm{Hz})$ & 7 & 8 & 9 \\
\hline 680 & 0 & 0 & 0 \\
1020 & 0 & 0 & 1 \\
1360 & 0 & 1 & 0 \\
1700 & 0 & 1 & 1 \\
2040 & 1 & 0 & 0 \\
2380 & 1 & 0 & 1 \\
2720 & 1 & 1 & 0 \\
3060 & 1 & 1 & 1 \\
\hline
\end{tabular}

Changes to DIP switches have no effect until the unit is reset. Shutdown and Test mode is entered when switches $4,5,6$ match $7,8,9$.

Figure 2A. Part locations and DIP switch. 


\section{J1 and J2 Modular Connector Pinouts}

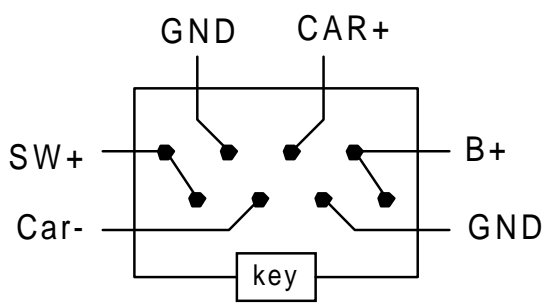

(solder side)

J1 Optional Ouput

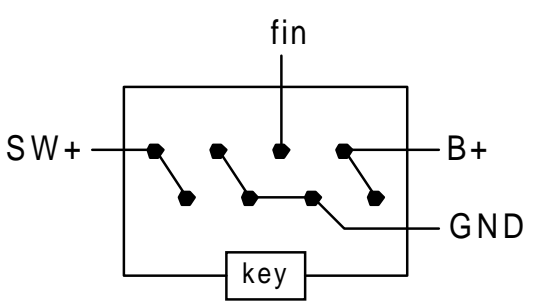

(solder side)

J2 Carrier input

Boards may be connected together via J1 and $\mathrm{J} 2$ to form multi-component instruments.

See Figure 2A for $1 / 0$ labels.

\section{$S$ and T Header Wiring}

T1

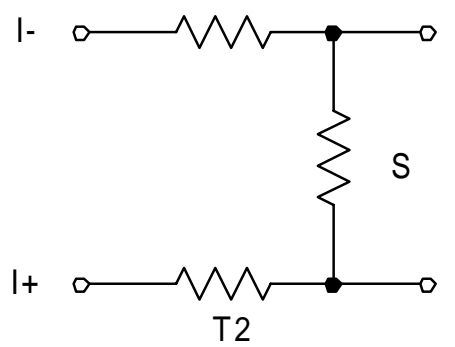

If the $S$ and $T$ pad is located off the board or T resistors are not used, then the T resistor positions must be jumpered to connect the seismometer to the input amplifier, U1.

\section{Test Points and Adjustments}

See Figure $2 \mathrm{~A}$ for test point and adjustment locations.

TP1 is the point to measure the preamplifier null in test mode.

TP2 shows the 0 volt referenced seismic signal.

TP3 shows the input to channel 1 of the ADC. It is biased at 2.5 volts.

TP4 shows the input to channel 2 of the ADC. It is biased at 2.5 volts.

TP5 shows the 11 subcarrier. Adjust this level with P5.

TP6 shows the f2 subcarrier. Adjust this level with P6.

P7 sets the output level of all subcarriers.

Do not adjust P1, P2, P3, P4 without using the Setup Procedure.

THESE ADJUSTMENTS ARE NOT USUALLY REQUIRED IN THE FIELD.

Figure 2B. Pinouts and test points. 


\section{Part II: Technical Reference}




\section{Circuit Description}

This description is intended for use with the schematic diagrams for McVCO included at the end of this section as Figure 3A, 3B, 3C. The description applies to printed circuit boards labeled "MCFM05" and "McVCO01". Integrated circuit packages shown in the diagrams are drawn in top view pin order with pin 1 on the upper left corner and the last pin on the upper right corner.

\section{Input/Output and DC Supply (Figure 3A)}

Drawn in the lower left of the schematic diagram are the solder pin connections for power and the output carrier frequency. They are labeled "SW+", "CAR+", "B+", "CAR-", and "GND".

$\mathbf{B}+$ is the connection to the positive side of a 12VDC battery or power supply.

GND is the negative connection to the supply and is ground, the 0 volt reference point. The schematic sometimes shows this connection with the ground symbol or indicates it with the "0V" label.

The positive side of the supply continues through the polarity-protection diode, D1, to the power switching circuits formed with U14, Q1, Q2, and Q3. The primary reason for power switching is to provide a clean reset for the microcontroller. In some circumstances, solar power systems can come up to the operating voltage gradually and fail to reset the microcontroller. This causes unpredictable behavior including the production of interfering output tones. A secondary use of the switch is to power an external load.

The primary switch is formed by U14, an op-amp connected as a comparator. The internal reference voltage at pin 2 is compared with a fraction of the positive supply at pin 3 and the output at pin 6 switches to the positive supply voltage $(+\mathrm{V})$ or ground. R35 provides positive feedback for hysteresis of one volt, so that the circuit switches $\mathrm{ON}(+\mathrm{V})$ with "B+" at 11.5 volts and OFF $(0 \mathrm{~V})$ when "B+" is about 10.5 volts.

For the MCFM05 version, U14's output splits going to the external load switch formed by Q2 and $\mathrm{Q} 3$, and to the voltage follower, Q1. The external load switch is brought out on "SW+". Care must be used when connecting external loads to minimize the resistance to the battery. If the battery voltage is low, voltage drops caused by IR losses can overcome the hysteresis of U14 and the switch will oscillate. The RC circuit in Q2's gate provides a delay in switching action while external loads stabilize.

In the McVCO01 version, the "SW+" output is switched by the microcontroller through Q2 and Q3. The switch turns on $10 \mathrm{~s}$ after power is turned on or the unit is reset. When "SW+" is 
switched on, if the battery voltage is low and IR losses in the wiring from the battery are greater than the hysteresis of the comparator, the comparator will switch off power. With the power off, the battery voltage recovers and McVCO turns on again. Ten seconds later the microcontroller turns on the load. Again, the battery voltage falls until it reaches the lower threshold of the comparator and the unit switches off. In this condition the subcarrier output will appear to be "frozen" at the channel center frequency and a very brief pulse will appear at "SW+". See Part III, "Using the Switched Power Output" for further comment.

The regulated power supply receives its input voltage from the emitter of Q1. This is approximately the output voltage of the U14 comparator until it exceeds the 15 VDC zener voltage of D2. Then the emitter of Q1 is clamped at 14.4 VDC. The zener clamp with the transient voltage suppressor, VS4, provide the overvoltage protection for the instrument. When 12 VDC solar panels are unloaded by batteries, voltage greater than $20 \mathrm{VDC}$ at reduced current is possible. VS4 clamps unloaded solar panels to about 20 VDC and can shunt $100 \mathrm{~mA}$ indefinitely. VS4 with VS1, VS2, VS3, VS5, VS6, and VS7 provide transient voltage protection on the instrument's inputs and outputs.

$\mathrm{U} 12$ is a +5 VDC regulator with an on chip comparator. The comparator is used to provide a reset pulse for the microcontroller. This reset is initiated by shorting across R40 on the pins provided. U13 is a switching inverter that converts the +5 VDC from U12 to the -5 VDC supply. These two supplies power the remaining circuits in the instrument.

The modulated audio carrier is output on the pins labeled "CAR+" and "CAR-" at up to $4 \mathrm{~V}$-p into $600 \Omega$ via the transformer, T1. C35 blocks external DC sources, R32 raises the primary impedance for a $600 \Omega$ impedance match, and with C21 isolates the output amplifier section of the quad op-amp, U3. The carrier output amp is a summing inverter whose gain varies from 2 to 1 with the adjustment of the Output Level pot, P7. The inputs to the summing amp are from the two onboard waveform generator outputs, f1 and f2 (see Figure 3C), and from an external audio carrier, $\mathrm{f}_{\mathrm{in}}$, which may be connected through $\mathrm{J} 2$.

J1 and J2 are modular jacks, shown on the left edge of Figure 3A. J1's connections are the same as the solder pin connections except for version MCFM05. For MCFM05, "B+" on the modular jacks is taken from the cathode of D1, the input polarity-protection diode. J2 provides unregulated power, ground, and a carrier input connection, $\mathrm{f}_{\mathrm{in}} . \mathrm{J} 1$ may be used to connect the instrument to a radio transmitter. $\mathrm{J} 2$ connects and powers other telemetered instruments. By using $\mathrm{J} 1$ and $\mathrm{J} 2$ together, a 3 component seismic station can be wired by chaining three McVCOs, see Part III, Three Component Connection, for more details.

Above $\mathrm{J} 2$, the diagram shows the seismometer input connection to the $\mathrm{S}$ and $\mathrm{T}$ Header. The seismometer coil leads are wired to "I+" and "I-" with the polarity indicated. The seismometer cable shield can be wired to the ground connection provided. From these solder pins the input goes to an 8-pin DIP socket where header mounted $\mathrm{S}$ and $\mathrm{T}$ resistors can be inserted. The T resistor is split into two equal $\mathrm{t} 1$ and $\mathrm{t} 2$ components for the sake of preserving input common- 
mode noise rejection. If $\mathrm{T}$ resistors are not used or are not located at the header, then shorting jumpers must replace $\mathrm{t} 1$ and $\mathrm{t} 2$ to complete the input connection. $\mathrm{R} 7$ connects the seismometer to a microcontroller I/O pin for pulse testing. From the header socket, connections are made to the seismic-signal amplifier shown in Figure $3 \boldsymbol{B}$.

The top of Figure $\mathbf{3 A}$ shows the components provided to support the optional second channel. These components and a small prototyping area next to U3 allow users to construct their own circuits, see Part III, Using the Low Gain Second Channel.

The USGS dual gain version of McVCO uses the optional components as shown in the center of Figure 3A. Here they form a fixed low gain interface from the filter (Figure 3B) to the second analog to digital converter channel (Figure 3C). This permits high and low gain measurements of the same seismic signal to be made and telemetered. The seismic signal is inverted by U3 at pin 1 and then level shifted by U15 to a 2.5 VDC bias appropriate for the 0 to 5 volt analog to digital converter (ADC) input. R48 is selected to attenuate the $10 \mathrm{~V}$ p-p swing at U3 pin 1 and to calibrate the overall gain of the channel. The $1.8 \mathrm{M} \Omega$ value shown gives the same frequency deviation as a USGS J502 with a deviation sensitivity of $115 \mathrm{~Hz} / 4.05 \mathrm{~V}$ operated at a gain of 40 $\mathrm{dB}$ (attenuation $=50 \mathrm{~dB}$ ).

\section{Seismic-signal Amplifier (Figure 3B)}

The signal from the $\mathrm{S}$ and $\mathrm{T}$ header socket (Figure $3 \boldsymbol{A}$ ) is applied to the instrumentation amplifier, $\mathrm{U} 1$, through the input circuit at In- and In+ (Figure 3B). Since the seismometer coil is not referenced to ground, R1 appears in series with R2 across the coil to give the standard $10 \mathrm{~K} \Omega$ input impedance. R3 and R4 are current limiting resistors that protect $\mathrm{U} 1$. Connections are shown for a relay, RL1, which is not used in this application. R5 and R6 reduce the gain of U1 and are not used in this application. The gain of U1 is 100 (40 dB).

A two-stage filter follows the input instrumentation amplifier. It is formed with two op-amps of the quad amplifier, U2. The 4-pole filter has a $30 \mathrm{~Hz}$ low-pass Butterworth response and is unity gain. Output from the filter at pin eight of $\mathrm{U} 2$ is available for low-gain second channel use and is the input to the variable-gain amplifier.

The variable-gain amplifier stage, shown at the bottom of Figure $\mathbf{3 B}$, is formed by the digital to analog converter (DAC), U4, and one section of U3, a quad op-amp. U4 is a 12 bit multiplying DAC and is used as the feedback element in the inverting amplifier made with U3. Through the serial interface lines "Data", "Clock" and "Gain" the microcontroller (Figure 3C) sets the amplification of this stage to provide the gain selected by the DIP switches. The Null control, P1 is used to zero the variable-gain amplifier at TP1 when the instrument is in shutdown mode (see Setup Procedure in Part I). The zero referenced output has a swing of almost $10 \mathrm{~V}$ p-p that must be reduced for the 0 to 5 volt analog to digital conversion. The Cal pot $\mathrm{P} 2$, provides the required attenuation and adjusts the instrument calibration (see Calibration Check in Part I). TP2 is the 
monitor test point where the amplified seismic signal can be safely viewed.

The bipolar output at TP2 must be level shifted to a 2.5 VDC bias for the ADC. This is done in the op-amp section of U5, where the signal is summed with a DC level from the Shift 1 pot, P3. At TP3, the seismic signal spans a 0 to 5 volt range referenced to $2.5 \mathrm{VDC}$ as required by the analog to digital conversion. The output of U5 on pin 6 connects to the first ADC channel on U6 pin 2 (CH0), see Figure 3C.

The microcontroller adjusts the offset of the seismic-signal amplifier as well as its gain. Another 12 bit DAC, U7, is interfaced to the controller through the serial interface lines "Data", "Clock" and "Offset" (see the center of Figure 3B). The reference section of U5 produces $200 \mathrm{mVDC}$ that is divided down to $50 \mathrm{mVDC}$ by R19 and R20 for the reference input of the DAC, U7 pin 1 . The DAC outputs a current at pin 3 that is a product of the DAC count and the voltage on the reference pin. At pin 14 of $\mathrm{U} 2$ the DAC current is changed to a voltage that is summed against the $50 \mathrm{mV}$ reference voltage. Consequently, the output of $\mathrm{U} 2$ on pin 1 is a voltage between $\pm 50 \mathrm{mV}$ which is proportional to DAC count. This voltage is applied to the ground reference terminal of the instrumentation amp, pin $1 \mathrm{U} 1$, and is used by the microcontroller to adjust the DC level at the seismic-signal amplifier output, U5 pin 6.

\section{ADC, Microcontroller, and Sinewave Generator (Figure 3C)}

The clock frequencies required by the microcontroller, U9, are derived from the $4.096 \mathrm{MHZ}$ crystal oscillator shown at the bottom of Figure 3C. The buffered output of the oscillator, U10 pin 10, drives a divider chain, U11, which produces $1.024 \mathrm{MHZ}$ at pin 7 for the microcontroller clock, U9 pin 27, and $1.0 \mathrm{KHz}$ at pin 1 for the real time clock input at U9 pin 1 . A $512 \mathrm{KHz}$ clock is produced by the divider on pin 6 for the sinewave generator clock inputs, pin 8 on U8 and U16.

Most of the microcontroller's activity is transmitted through the serial interface formed with its I/O pins. Common lines for serial clock, U9 pin 13, and serial data, U9 pin 14, connect the seismic-signal amplifier gain and offset DACs, the ADC, and the f1 and $\mathrm{f} 2$ waveform generators. Individual chip select lines address each IC as shown.

The DIP switch, DS1 is read on microcontroller I/O pins 17 to 25 . Switch 1 is connected to pin 25 with switch 9 on pin 17. The table on the right side of Figure $3 C$ shows how gain and frequency are selected by the switches. The switches are "active low", an ON switch pulls the line low which the microcontroller interprets as a logic 0 . The invalid condition of $\mathrm{f} 1$ and $\mathrm{f} 2$ selected for the same frequency, results in a shutdown of the instrument. This condition is used for adjusting the seismic-signal amplifier (see Part I, Setup Procedure) but must be avoided when the instrument is in use, even if the second channel frequency, f2 is not used. DS1 is read by the microcontroller only after a power cycle or reset. 
The four remaining I/O lines are used for diverse purposes. The line on pin 6 connects to the coil of the seismic-signal amplifier input relay, RL1 which is not used in this application. Pin 7 allows a pulse to be applied to the seismometer through R7 (Figure $3 A$ ) as part of a programmed periodic test. The I/O lines on pins 15 and 16 are used as program branch flags in some versions of the microcontroller program where $5 \mathrm{~V}$ on pin 15 enables the periodic test routine and $5 \mathrm{~V}$ on pin 16 enables two channel operation.

The analog to digital converter (ADC), U6, responds to requests from the microcontroller for a conversion of the input voltage on either of the two input channels at pin 2 or 3 . After the conversion is transferred to the microcontroller, the ADC idles while the controller changes the measurement into a code and sends it to the f1 or f2 waveform generator, U8 or U16. The generator uses the code to synthesize the instantaneous output frequency corresponding to the ADC measurement and the selected carrier frequency. The cycle begins again with a new ADC measurement and repeats approximately 660 times per second for a single channel and about half that rate for two channel operation.

The f1 Level pot, P5, and the f2 Level pot, P6, adjust the output amplitudes of the waveform generators, U8 and U16, at TP5 and TP6. The modulated carriers at these test points are connected to the output summing amp, see Figure $\mathbf{3 A}$.

During the measurement cycle the microcontroller's real time clock is examined. At the correct interval an adjustment is made to the offset DAC (Figure 3B) and the 24 or $18 \mathrm{hr}$ timer registers are incremented and checked for "timeout". If selected, at "timeout" the programed test routine is entered. At the end of the test routine the measurement cycle resumes.

At power up or after a reset, the instrument goes into an autozero mode. At this time, f1 and f2 are set to their carrier center frequencies while offset is removed from the seismic-signal amplifier output by adjusting the count on the offset DAC. The autozero lasts for 10 seconds.

With the McVCO01 version of the instrument, the programmed test routine is executed immediately after the autozero. The routine is about 42 seconds long and consists of $10.25 \mathrm{~s}$ of $21.25 \mathrm{~Hz}$ modulation of the subcarrier, followed by $18 \mathrm{~s}$ for the seismometer pulse test, and then by $13 \mathrm{~s}$ of pulses which give the gain setting and ID of the instrument. Where a positive pulse is "1" and a negative pulse is "0", the first three pulses give the gain DIP switch setting at the last reset when the gain was set. The last 10 pulses are the binary representation of the unique ID number programmed into the microcontroller. See Part III, "McVCO Test Cycle" for further details.

\section{Schematic Diagrams: Figure 3}

The following three pages contain McVCO's schematic diagrams. 
Part II: Technical Reference

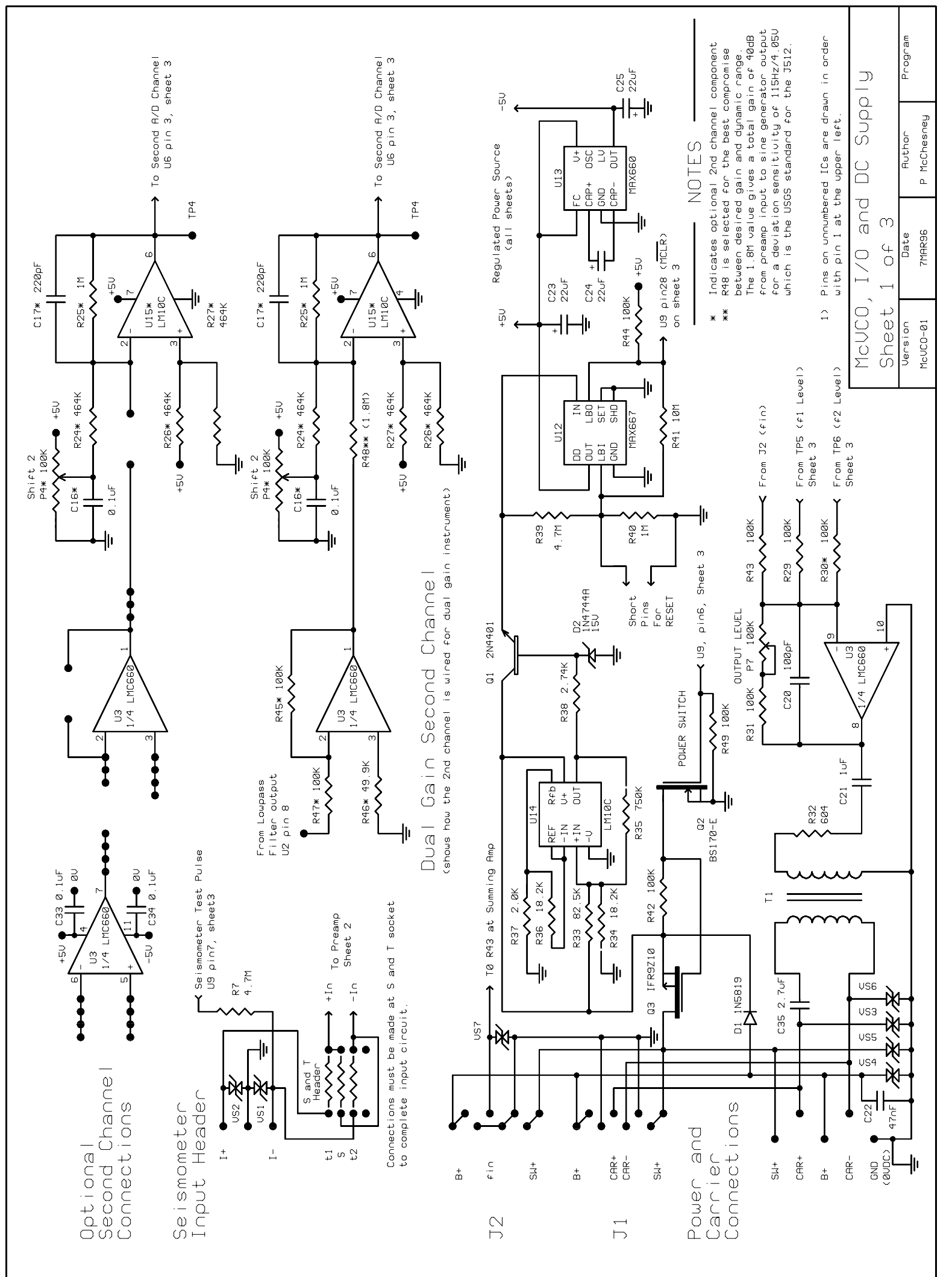

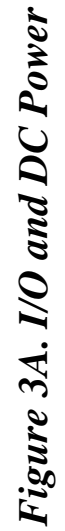


Part II: Technical Reference

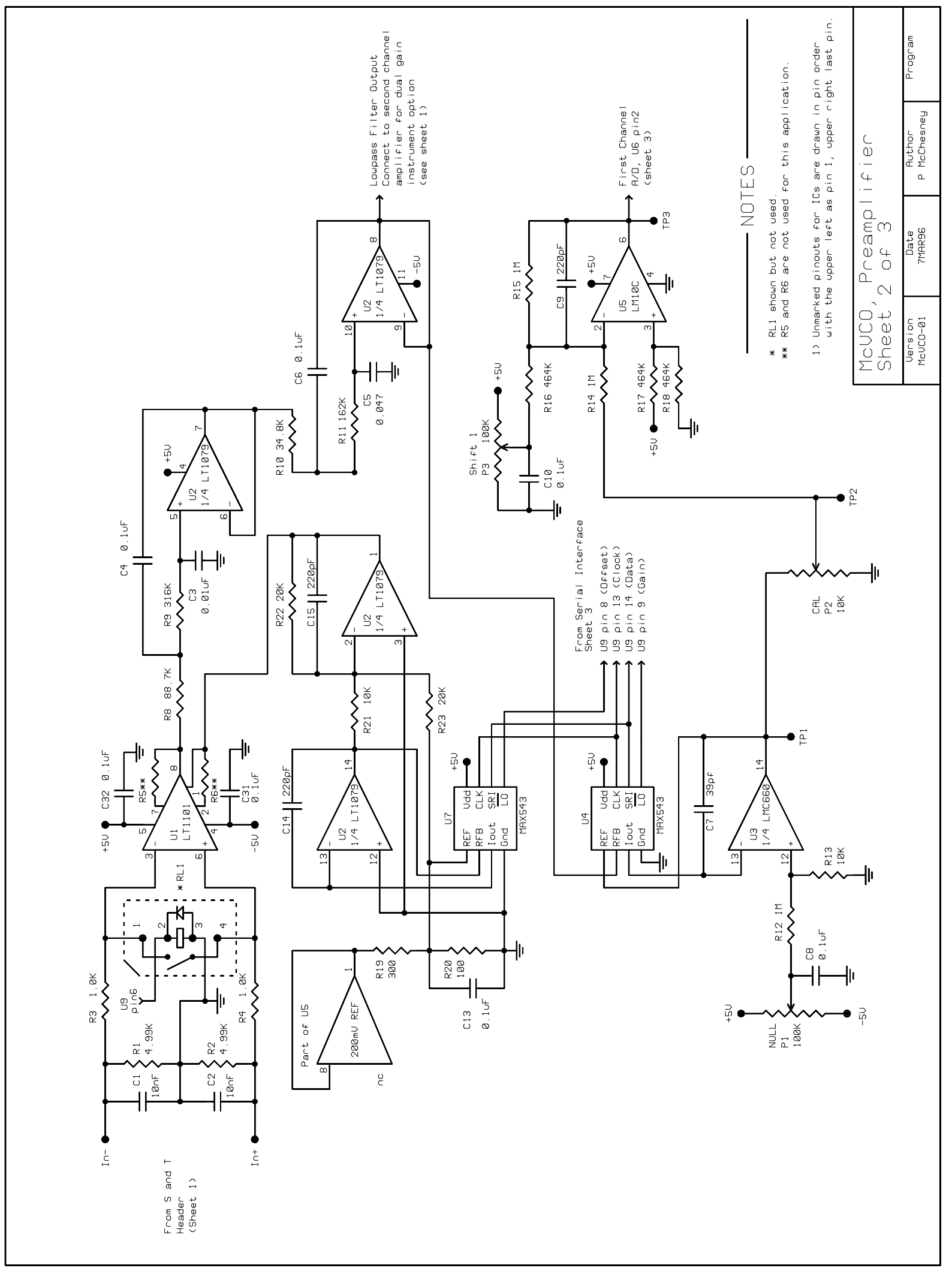

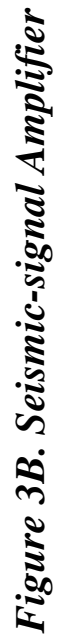


Part II: Technical Reference

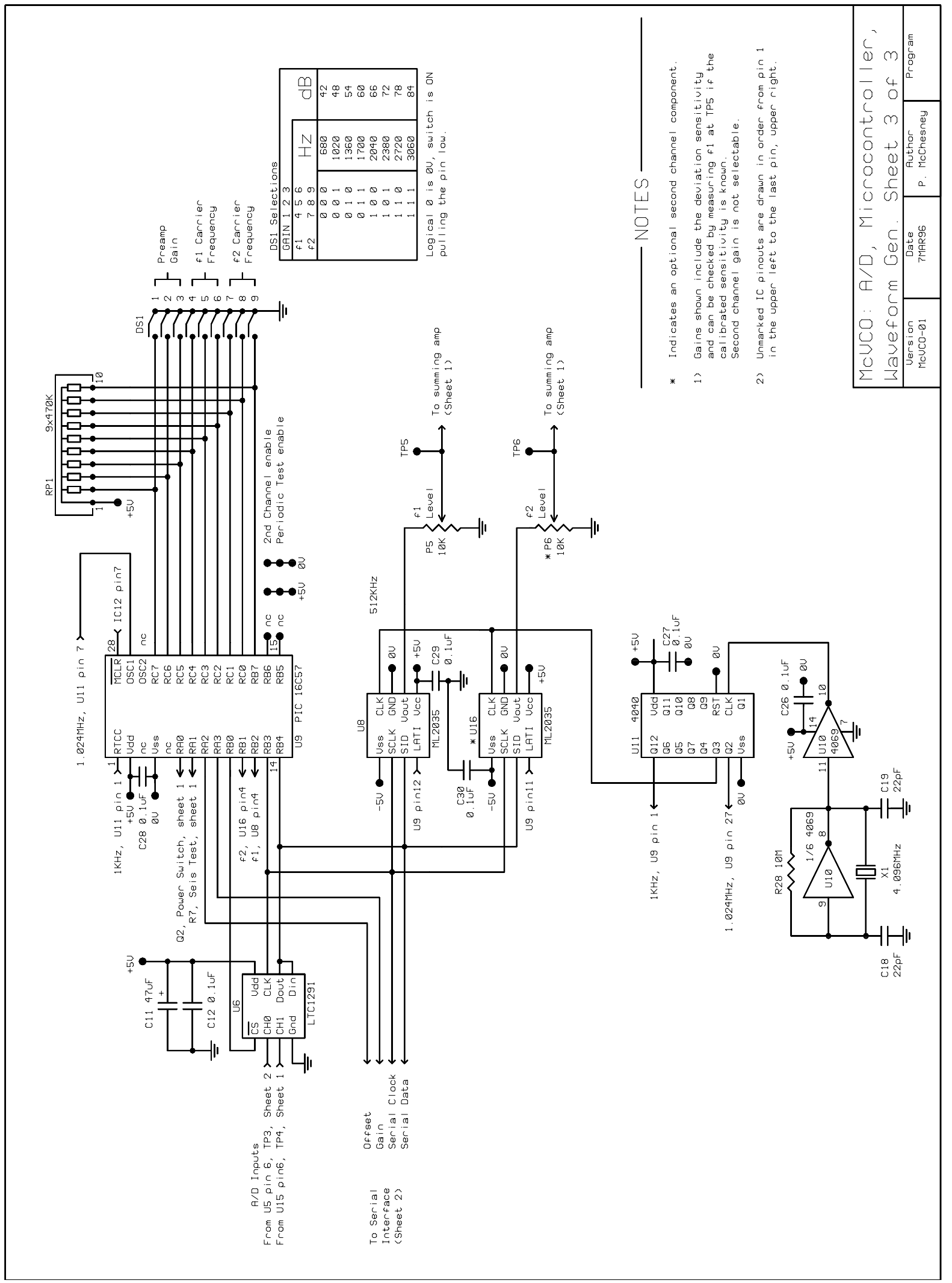

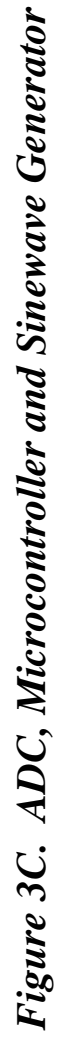




\section{Specifications}

These specifications, where they are not inherent to the design, are typical of the instrument performance. They are an attempt to fairly characterize the instrument in spite of limited testing capacity. Comment from users is welcome and revisions to the specifications will be made where they are in error.

\section{$\underline{\text { Seismic-signal amplifier }}$}

Input Type

Gain Adjustment

Gain accuracy

Frequency Response

Output noise level

Sensitivity

Second Channel Amplifier
Differential, with $\mathrm{Z}_{\text {in }}=10 \mathrm{~K} \Omega$ for $\mathrm{f}<30 \mathrm{~Hz}$.

Connections for $\mathrm{S}$ and $\mathrm{T}$ resistors provided.

$42 \mathrm{~dB}$ in eight switch selected settings $6 \mathrm{~dB}$ apart. Usually starting at $42 \mathrm{~dB}$ with a maximum setting of $84 \mathrm{~dB}$.

Better than $3 \%$ across all ranges.

Low-pass, 4-pole, $30 \mathrm{~Hz}$ Butterworth filter (-24 dB/octave above $30 \mathrm{~Hz}$ ). The amplifier is direct coupled but DC performance is affected by offset control. Attenuation by offset control circuit less than $3 \%$ for input signals with slew rates greater than $50 \mathrm{nV} / \mathrm{s}$ and less than $1 \%$ for slew rates greater than $100 \mathrm{nV} / \mathrm{s}$. See discussion in Part III, Gain and Frequency Response.

$\mathrm{Vn}($ out $)=3 \mathrm{mV}$ rms at maximum gain, decreasing proportionally with gain. Equivalent to $20 \mathrm{mV}$ p-p at the ADC input or $0.4 \%$ of ADC span for \pm 8 ADC counts.

$2.6 \mu \mathrm{V} \mathrm{rms}$ at the input with maximum gain produces $35 \mathrm{mV} \mathrm{rms}$ at the ADC (100 mV p-p) with $3 \mathrm{mV}$ rms noise for $\mathrm{SNR}=21.3 \mathrm{~dB}$ when the gain tables are set for a deviation sensitivity of 41.67 $\mathrm{Hz} / \mathrm{V}$. Other deviation sensitivities are similar. See discussion in Part III, Gain and Frequency Response.

User defined. The gain is $40 \mathrm{~dB}$ for USGS dual gain versions. 


\section{Modulator}

Output Range

Deviation Sensitivity

Analog to Digital

Conversion (ADC)

Frequency Resolution

Center Frequencies

Frequency Stability

Output Type

Output Amplifier

Output Level

Noise + Distortion $\pm 125 \mathrm{~Hz}$ from center frequency, absolutely limited because the instantaneous frequency is calculated from the analog to digital conversion (ADC) which exactly spans this range.

$($ Output Range $) /($ ADC Span $)=(250 \mathrm{~Hz}) /(5 \mathrm{~V})$ Other sensitivities are emulated by scaling gain in the seismic signal amplifier. See discussion in Part III, Gain and Frequency Response.

12 bit successive approximation ADC sampling at 660 sps for single channel operation and 330 sps for two channels.

The sinewave generator minimum frequency change is $250 \mathrm{~Hz} / 4096$ and fully resolves the 12 bit ADC.

\section{$\underline{\text { Audio Carrier Out }}$}

Any of the following center frequencies are switch selectable for both channels.

$680,1020,1360,1700,2040,2380,2720,3060 \mathrm{~Hz}$

The sinewave generator output is crystal derived so the main factor in off center operation is seismic-signal amplifier output offset. This offset is actively regulated by the microcontroller so that the output frequency is within $\pm 2 \mathrm{~Hz}$ of center frequency after autozero on reset and adjusts to within $1 \mathrm{~Hz}$ long term.

Transformer coupled with a DC blocking capacitor. The secondary floats and has a reflected differential output impedance of $600 \Omega$.

Output amplifier is a summing inverter with provision for a second channel carrier and the import of an external carrier.

0 to $4 \mathrm{~V}$ p-p into $600 \Omega(2.6 \mathrm{dBm} \max )$.

Better than $45 \mathrm{~dB}$ down relative to the carrier at full power into 600 $\Omega$. Better than $47 \mathrm{~dB}$ down relative to the carrier at $1 \mathrm{~V}$ p-p into 10 $\mathrm{K} \Omega$. 


\section{Power and Environmental}

Supply Voltage Range

Power Consumption

Protection

Operating Temperature

Range

Board Size
11 to 20 VDC. Can withstand the unloaded output of a $12 \mathrm{~V}, 30 \mathrm{~W}$ solar panel indefinitely (approximately $20 \mathrm{~V}$ shunt regulated by power input TVS). An internal power switch assures microcontroller reset, $\mathrm{ON}$ at $11.5 \mathrm{~V}, \mathrm{OFF}$ at $10.5 \mathrm{~V}$.

Single channel, $220 \mathrm{~mW}$ at $12 \mathrm{VDC}(18 \mathrm{~mA})$

Two channel, $300 \mathrm{~mW}$ at $12 \mathrm{VDC}$ (25 mA).

All inputs and outputs protected by $15 \mathrm{~V}$ transient voltage suppressors (TVS) except the power input TVS rated at $20 \mathrm{~V}$. The power supply is reverse-polarity protected by a series diode.

Units with commercial grade parts have tested successfully to $-40^{\circ} \mathrm{C}$. Potentially destructive high temperature tests have not been conducted. The suggested range is -30 to $+50^{\circ} \mathrm{C}$ based on the operating temperature of commercial grade parts.

$\mathrm{L}=7.7 ", \mathrm{~W}=3.9^{\prime \prime}, \mathrm{H}=1.0 "(\max )$ 


\section{Part III: Application Notes}




\section{Gain and Frequency Response}

When McVCO was designed, it was hoped that it would be possible to say that McVCO was a digital imitation of a particular VCO and to copy its response. The situation was not that simple. Not only did the VCOs vary, but they were often modified by users. Consequently, McVCO has its own response characteristics and the design, rather than imitating another instrument, tries to use microcontroller technology to best advantage. Unfortunately this requires the user to learn something about how McVCO mimics an analog VCO. Not everyone will wish to view the details, so a short summary of gain and frequency responses is given followed by more detailed discussions.

\section{Gain Response}

The gain of McVCO is referenced to the signal at the seismic-signal amplifier input. It does not include a term for any attenuation of the seismic signal that may occur in $\mathrm{S}$ and $\mathrm{T}$ resistors between the seismometer and the amplifier. The issue of damping and seismometer calibration is left to the user. The input impedance that the seismic-signal amplifier presents to the $\mathrm{S}$ and $\mathrm{T}$ network is $10 \mathrm{~K} \Omega$ and must be included in $\mathrm{S}$ and $\mathrm{T}$ calculations.

The electronic gain of the seismic-signal amplifier is adjusted to give an effective deviation sensitivity so that:

$$
\Delta \mathbf{f}=\left(\mathbf{S}_{\mathrm{in}}\right) \times\left(\mathrm{A}_{\text {DIP }}\right) \times(\mathrm{EDS})
$$

where: $\quad \Delta \mathrm{f}$ is the change in output frequency $(\mathrm{Hz})$.

$\mathrm{S}_{\text {in }}$ is the seismic-signal amplifier input signal (V).

$\mathrm{A}_{\mathrm{DIP}}$ is the DIP switch selected gain (V/V).

EDS is the effective deviation sensitivity $(\mathrm{Hz} / \mathrm{V})$.

The effective deviation sensitivity is the calibrated deviation sensitivity specified by the user for a particular system. Adjusting the Cal pot for other deviation sensitivities will sacrifice dynamic range due to clipping at the analog to digital converter (ADC) or clipping in the seismic-signal amplifier.

\section{Gain Response Discussion}

The issue of an effective deviation sensitivity arises because the real deviation sensitivity of the instrument is fixed by the ADC input span of 0 to 5 volts and the channel bandwidth of $250 \mathrm{~Hz}$. Departures from this $50 \mathrm{~Hz} /$ volt sensitivity can only be carried out by adjusting gain ahead of the ADC. 
Part III: Application Notes

McVCO has programmable gain and uses it to mimic other deviation sensitivities. When the microcontroller is programmed, its internal gain tables are written for a particular calibrated deviation sensitivity. It is possible to adjust $\mathrm{P} 2$, the $\mathrm{Cal}$ pot for other calibrations but doing this will decrease the dynamic range of the seismic-signal amplifier because the Cal pot provides attenuation from the $10 \mathrm{~V}$ p-p output of the programmable gain amplifier to the $5 \mathrm{~V}$-p $\mathrm{p}$ input of the ADC.

Maximum dynamic range is achieved when the $10 \mathrm{~V}$ p-p output swings full span while the ADC input swings full span. Allowing for the fact that the amplifiers involved do not swing the full supply and for some offset, the gain of the Cal pot for maximum dynamic range is:

$$
\mathrm{v}_{\text {out }} / \mathrm{v}_{\text {in }}=4.9 \mathrm{v} / 9.5 \mathrm{v}=0.5158
$$

This is the "sweet spot" of the Cal pot where ideally both calibration and maximum dynamic range are found. The pot is provided to trim gain errors in the seismic-signal amplifier while maintaining full dynamic range and not as a means to change the calibration.

Suppose recalibration is required. As an example, the Pacific Northwest Seismographic Network (PNSN) uses a calibrated deviation sensitivity of $41.67 \mathrm{~Hz} /$ volt and a common USGS value is $28.40 \mathrm{~Hz} /$ volt. How much dynamic range is lost by recalibrating the PNSN instrument as a USGS instrument with the Cal pot?

When the calibration is changed, the gain of the Cal pot is reduced from 0.5158 to 0.3515 . This means that a full swing output of the programmable gain amplifier produces $0.3515 \times 9.5 \mathrm{~V} p$-p at the ADC input or $3.34 \mathrm{~V}$ p-p instead of the full span of $5 \mathrm{~V} \mathrm{p}$-p. The loss in dynamic range is $20 \log (3.34 / 5)=-3.5 \mathrm{~dB}$. This may not be too bad, but the best way to recalibrate is to install a microcontroller with the correct gain tables if strong signals are anticipated. See the Introduction for a source of programmed microcontrollers.

If large changes in gain are wanted, $20 \mathrm{~dB}$ can be subtracted from the input instrumentation amplifier by installing shorting jumpers in the circuit positions marked R5 and R6 near U1. Other values of gain can be achieved with resistors installed here. See Linear Technology's data sheet for the LT1101. Increasing gain beyond the approximately $90 \mathrm{~dB}$ obtainable with the Cal pot at maximum is not a good idea because of inherent input amplifier noise.

The McVCO's $10 \mathrm{~K} \Omega$ input impedance is due to the series combination of R1 and R2. Another pair of equal value resistors can be used to change the impedance. They should be $1 \%$ metal film resistors so that the common mode rejection ratio (CMRR) is preserved and noise is reduced.

There is a second channel on McVCO for those who wish to do their own sensor interfacing. See "Using the Low Gain Second Channel" in Part III of this handbook. 


\section{Frequency Response}

McVCO's upper frequency roll off is determined by a four-pole active Butterworth filter. The -3 $\mathrm{dB}$ breakpoint of the filter is at $30 \mathrm{~Hz}$ and the attenuation increases at $24 \mathrm{~dB}$ /octave.

There is no lower frequency cutoff. At some combination of amplitude and frequency, the feedback loop that produces the offset correction for the seismic-signal amplifier will start to attenuate and distort a very long period signal. The size of this correction is below the noise level of the input amplifier and is made at 32 second intervals. Consequently its effect on signals with periods less than $32 \mathrm{~s}$ is undetectable. The offset correction scheme does prevent the seismicsignal amplifier from functioning as a DC amplifier despite the fact that it is directly coupled.

\section{Frequency Response Discussion}

Though McVCO is analog in and analog out, at its core it is a sampled data system. In two channel mode the sample rate is 330 samples/sec and in single channel mode it is a little over twice this. This gives the low-pass filter two jobs. It must reduce noise by limiting the bandwidth to the frequencies of interest and prevent noise beyond half the sample rate from aliasing into the passband. The attenuation at half the sample rate for the two channel mode is about $60 \mathrm{~dB}$ and for the single channel mode is greater than $80 \mathrm{~dB}$. The Butterworth filter's phase response in the passband is not linear but it is standard and discussed widely in the literature.

What happens to McVCO's response at the low end of its frequency response is much less conventional. There is no high-pass filter, the seismic-signal amplifier is direct coupled. However, very long period signals are distorted and attenuated by the offset correction circuit.

The principle behind the offset correction scheme is simple. Seismic signals over time have an average value of zero and offset voltages do not. Consequently it is possible to cancel offsets and preserve signal by slowly adjusting the voltage through a feedback loop to maintain an average value of zero at the input to the ADC.

McVCO carries out this scheme by keeping a 12 bit offset count in the digital to analog converter (DAC), U7. This count produces a voltage that is summed with the signal in the output of U1, the first stage of the seismic-signal amplifier. The polarity of the voltage summed at U1 is that required to cancel the voltage at the input of the ADC. Every 32 seconds, the microcontroller looks at the most recent ADC and increases or decreases the offset count so that the ADC input is pulled toward its zero signal value (the center of the ADC input span is about 2.5 VDC). 
Part III: Application Notes

The components of the voltage at the ADC input are seismic signal, noise, and the seismic-signal amplifier offset. Any particular adjustment to the digital to analog converter (DAC) count may be a response to signal or noise and be wrong in terms of canceling offset. However, over many offset correction cycles, signal and noise with average values of zero during the 32 s correction period will disappear from the DAC count. The DAC count will contain offset and a fraction of any signal components with periods longer than $32 \mathrm{~s}$. It is this offset and part of the long period signal that is removed from the input signal at the output of $U 1$.

Though the offset correction circuit attenuates signals with periods longer than $32 \mathrm{~s}$, McVCO can successfully measure signals with periods longer than this. However, before discussing these longer period signals, it is best to finish discussing signals with periods shorter than the offset correction period.

Signals with periods less than $32 \mathrm{~s}$ see at most one correction. The size of any correction is \pm 1 DAC count. The correction circuit output voltage span is $\pm 50 \mathrm{mV}$. Since there are 4096 total DAC counts, a change of one count is a change of $24.4 \mu \mathrm{V}$. This single count voltage is not amplified by the $40 \mathrm{~dB}$ gain of the input stage and therefore corresponds to $0.244 \mu \mathrm{V}$ at the input to U1, an LT1101. Linear Technology says that the typical 0.1 to $10 \mathrm{~Hz}$ noise of the LT1101 is 0.9 $\mu \mathrm{V}$ p-p. A single count change is less than $1 / 3$ this noise level and is virtually invisible for signals with periods shorter than the $32 \mathrm{~s}$ update period of the offset count.

For signals with periods longer than $32 \mathrm{~s}$, the situation is more complicated. The question is how many offset corrections can a signal tolerate before it is no longer useable? The change in the signal depends on both the signal period and amplitude. Large signals will change proportionately less than small ones and longer period signals will see more offset corrections during each cycle than shorter period signals. This product of amplitude and frequency describes the slew rate (S) of a sinewave and where $\mathrm{A}$ is the peak to peak amplitude and $\mathrm{P}$ is the period,

$$
\mathbf{S}=\mathbf{A}_{(\mathbf{p}-\mathbf{p})} \times \mathbf{p i} / \mathbf{P}
$$

The action of the offset correction feedback loop is always to pull the ADC input toward the zero signal level. It opposes the input signal as it moves away from zero and aids the signal as it moves to zero. This action attenuates the input signal and the effect of the offset correction circuit on long period signals can be partially described as an attenuation that varies with the signal slew rate. Figure 4 shows this relation.

The curve of Figure 4 shows a gradual change in the attenuation of signals with slew rates greater than $50 \mathrm{nV} / \mathrm{s}$. It shows a rapid increase in attenuation for signals with slew rates slower than this. Consequently, it is of interest to look at McVCO performance for signals with this slew rate. 


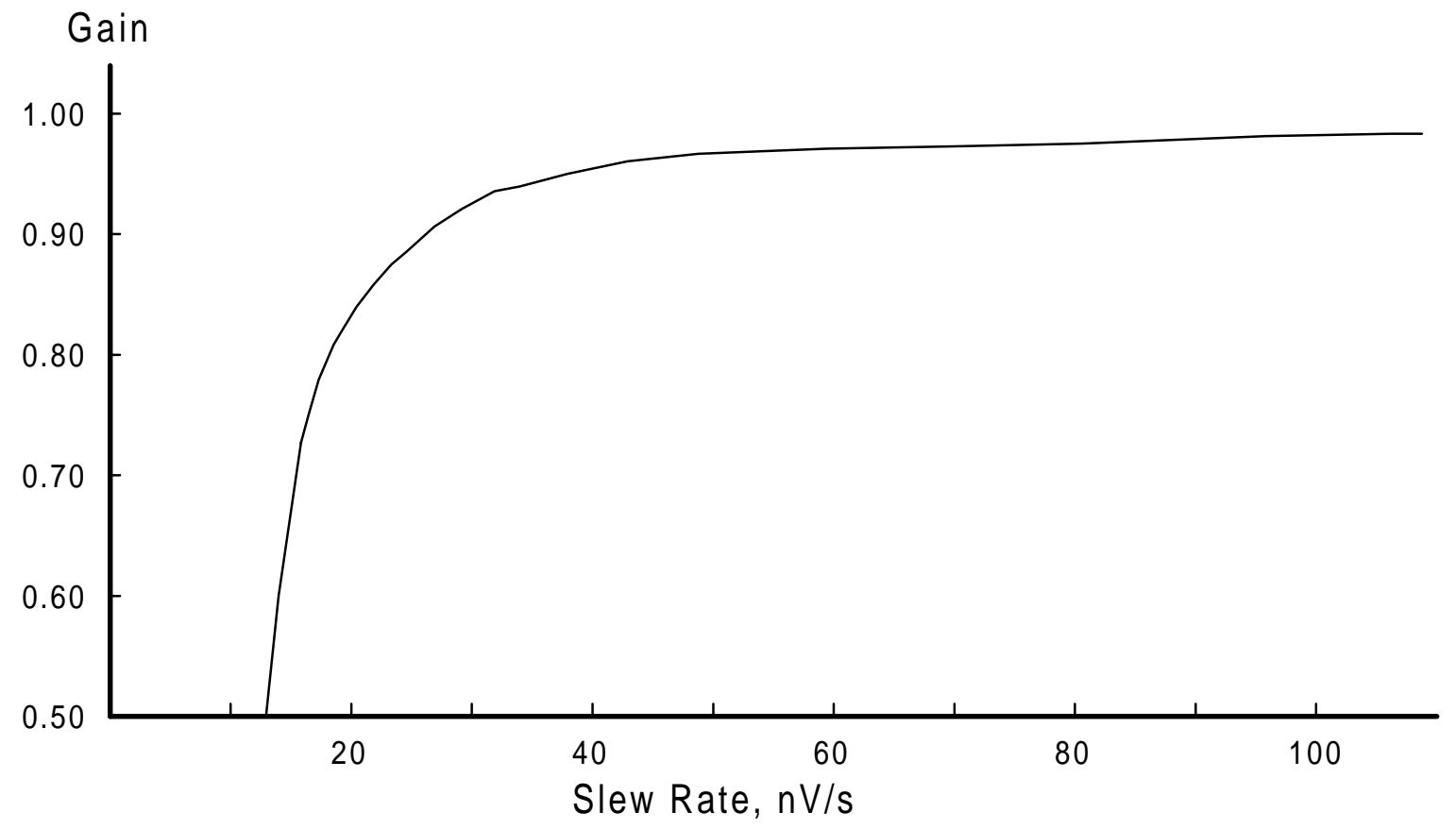

Figure 4. The effect of the offset correction on signal amplitude as the signal slew rate changes. The attenuation is expressed as gain (Vout / Vin) normalized to one in comparison to an unattenuated signal with a much higher slew rate.

$\mathrm{McVCO}$ is specified to have a greater than $20 \mathrm{~dB}$ signal to noise ratio at the $\mathrm{ADC}$ for a signal of $2.6 \mu \mathrm{V} \mathrm{rms}(7.35 \mu \mathrm{Vp}-\mathrm{p})$ at maximum gain. The period of a signal with this amplitude and a slew rate of $50 \mathrm{nV} / \mathrm{s}$ is $460 \mathrm{~s}$. If the approximately $3 \%$ attenuation at $50 \mathrm{nV} / \mathrm{s}$ is acceptable, then this can be taken as the smallest amplitude and longest period signal that McVCO can measure without interference from the offset correction circuit. If larger signals with faster slew rates are of interest, then McVCO can measure signals with longer periods than $460 \mathrm{~s}$.

Attenuation is not the only effect of the offset correction circuit on the signal. As the slew rate decreases to $25 \mathrm{nV} / \mathrm{s}$, distortion becomes clearly visible in the signal at the ADC. The distortion in the signal comes from the offset correction waveform. It is a positive or negative going ramp. During the positive half cycle of the signal at the ADC, the correction circuit steps negatively one count every $32 \mathrm{~s}$, while for the negative half cycle it steps positively at the same rate. This ramp is summed with the input signal at the output of U1. When the slew rate decreases, the ramp becomes larger and distortion increases. Figure 5 shows the distortion of a signal with a slew rate of $16 \mathrm{nV} / \mathrm{s}$. This signal has a normalized gain of about 0.7 . 


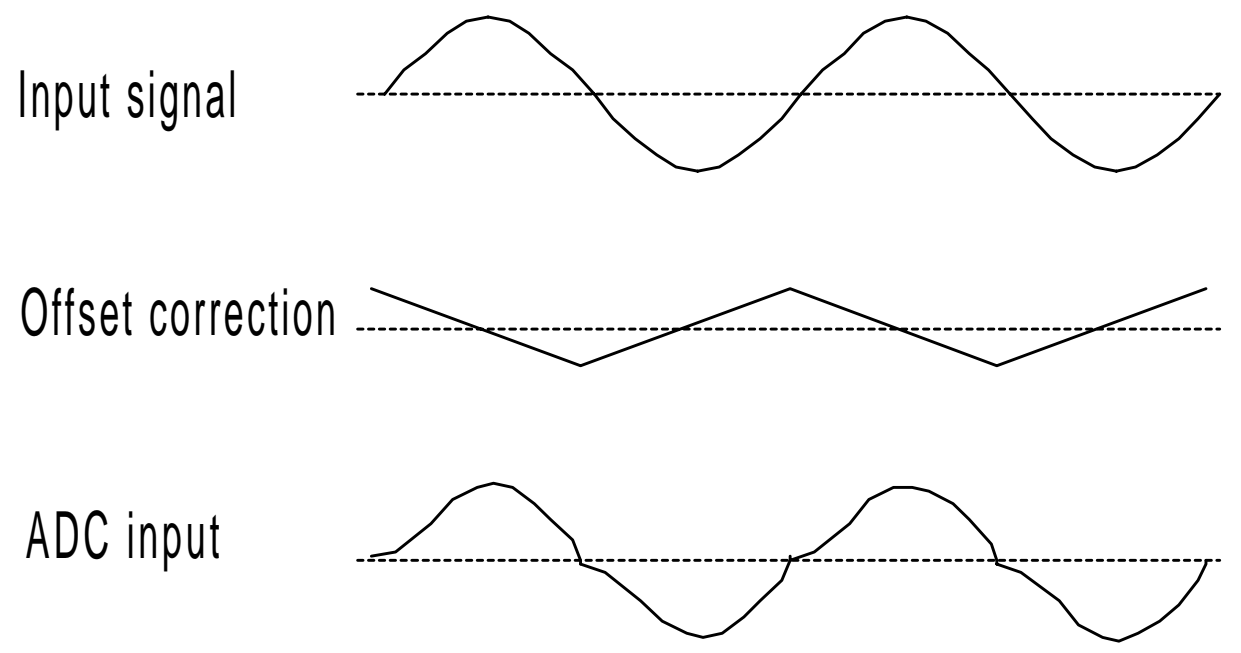

Figure 5. The distortion of a $16 \mathrm{nV/s}$ signal caused by the offset correction circuit. The drawing is not to scale. The ADC signal is 0.7 times the amplitude of an unattenuated signal with a faster slew rate.

Though the offset correction circuit produces an unconventional low frequency response, McVCO can effectively measure long period signals if they have slew rates greater than $50 \mathrm{nV} / \mathrm{s}$. If operation to DC is required, McVCO's second channel is outside the correction loop. See Part III, Using the Low Gain Second Channel. 


\section{Using the Second Channel}

Most McVCOs supplied so far are configured for dual gain operation where a high-gain first channel is supplemented by a low-gain second channel. The second channel is connected to the first channel low-pass filter output and shares the same signal. The purpose of this configuration is to increase the dynamic range of the instrument in the presence of strong input signals. Users of the instrument may wish to change this mode of operation for two reasons. There may not be space in the telemetry network for a second channel, or the second channel may be required for a different purpose. Defeating the second channel and the interfacing of other signals are discussed below.

\section{Turning Off the Second Channel}

The second channel is most easily turned off by removing the second channel sinewave generator, U16, from the circuit and grounding second channel input to the output summing amp by turning the f2 Level control, P6, fully counterclockwise. This crude method has the advantage of saving about $7 \mathrm{~mA}$ and prevents the second channel from ever spilling unwanted signal or noise into the telemetry network. The ML2035 sinewave generator draws $2 \mathrm{~mA}$ in the processor controlled power down mode so pulling the chip saves more power than turning it off with the microcontroller. A further $0.5 \mathrm{~mA}$ can be saved by removing the second channel level shift amplifier, U15.

There is a way of telling the microcontroller that single channel mode is required. If pin 16 of U9, the microcontroller is held low, the controller will not request a second channel sample from the analog to digital converter (ADC). This will double the sample rate for the first channel. Increasing the sample rate increases the effectiveness of the anti-aliasing function of the first channel low-pass filter. The attenuation of this filter at half the sample rate during two channel operation is $60 \mathrm{~dB}$. This increases to $80 \mathrm{~dB}$ for single channel operation only.

The increased attenuation is an advantage if there are noise sources with frequency components above half the sample rate. The sample rate for two channel operation is $330 \mathrm{samples} / \mathrm{s}$. The single channel rate is about 660 samples/s. Raising the noise floor with aliased noise reduces the instrument's ability to transmit small signals but other noise sources in the telemetry path are usually more significant in reducing overall sensitivity than the change from one to two channel operation.

If operating only the second channel is necessary, then the first channel may be disabled in a similar way by removing U8 and U5. There is no way to tell the processor to sample only the second channel so pin 16 of U9 should be tied high $(5 \mathrm{~V})$ for all second channel measurements except for those which are made during the test cycle (see Part III, Monitoring Site Battery Voltage). 


\section{Interfacing to the Second Channel}

This is a rough guide to using the second channel intended for those who have some experience with analog circuit design. Specific applications cannot be covered, so the focus is on the general characteristics of the second channel.

The schematic diagram (Figure $3 \boldsymbol{A}$ ) shows the second channel in two ways. The middle of the page shows a low gain second channel wired to the first channel low-pass filter output. Above this some of the same components are shown unconnected. These components are available for do-ityourself second channel interfacing and there is a small prototyping area near U3 for this purpose.

The two uncommitted U3 op amps may or may not suit a particular application. The LMC660 quad amplifier is not a precision DC amplifier and is probably not suitable for high gain DC circuits. It was picked because its rail to rail output swing made it useful in the variable-gain amplifier circuit while the $1.1 \mathrm{MHz}$ gain-bandwidth product, low distortion and $600 \Omega$ drive capability make it a good choice for the output summing amplifier. Finding these characteristics in a low power amplifier is difficult. Substituting another quad op amp for the LMC660 at U3 is not recommended. Consult the LMC660 data sheet for full specifications.

Whether or not the onboard op amps are used, the entry point to the second channel is through the level shifter, U15. This circuit shifts signals to the $2.5 \mathrm{VDC}$ center of the 0 to $5 \mathrm{~V}$ span of the ADC. The circuit is a summing inverter biased to $2.5 \mathrm{VDC}$ at the noninverting input. The summing point sees input from $\mathrm{P} 4$ and the signal through gain-setting input resistors. These resistors are picked so that with the maximum input signal present, $\mathrm{P} 4$ can be adjusted for a centered full span signal swing at the ADC.

Unlike a conventional VCO designed with analog components, McVCO has a fixed modulator sensitivity. The 5 volt analog to digital converter (ADC) input span corresponds exactly to a \pm 125 $\mathrm{Hz}$ deviation from center frequency for a constant modulator sensitivity of $50 \mathrm{~Hz} / \mathrm{V}$. It is often required to operate with a deviation sensitivity different from this modulator sensitivity. This can be achieved by changing the gain ahead of the modulator so that the modulator responds to an input signal at the seismic-signal amplifier as if it had a different deviation sensitivity.

Unfortunately this can be confusing. Once the modulator sensitivity and the deviation sensitivity are no longer the same, the calibrated gain setting of the instrument differs from the actual signal gain. Suppose that it is desired to operate McVCO's second channel at a calibrated gain of 100 $(40 \mathrm{~dB})$ in a system that is set up for a deviation sensitivity of $115 \mathrm{~Hz} / 4.05 \mathrm{~V}$. McVCO's modulator sensitivity $(50 \mathrm{~Hz} / \mathrm{V})$ is too sensitive and the gain of the seismic-signal amplifier must be reduced proportionately. 
The signal gain from the amplifier $\left(\mathrm{A}_{\text {signal }}\right)$ is

$$
\mathbf{A}_{\text {signal }}=\mathbf{A}_{\text {cal }} \times \mathbf{D}_{\text {sens }} / \mathbf{M}_{\text {sens }}
$$

where $\quad A_{\text {cal }}$ is the desired calibrated gain $(\mathrm{V} / \mathrm{V})$.

$\mathrm{D}_{\text {sens }}$ is the deviation sensitivity of the system $(\mathrm{Hz} / \mathrm{V})$.

$\mathrm{M}_{\text {sens }}$ is the McVCO's modulator sensitivity $(50 \mathrm{~Hz} / \mathrm{V})$.

In this case, the seismic-signal amplifier signal gain must be set to 56.79 so that McVCO mimics an instrument with the required deviation sensitivity set for a gain of 100. Each time McVCO is used in a system with a deviation sensitivity that differs from its modulator sensitivity, the amplifier signal gain must be scaled to compensate. See Part III "McVCO Gain and Frequency Response" for a related discussion.

When interfacing a signal to the second channel it is important to be aware that McVCO is a sampled data system and cannot process signals above half the sample rate. Signal and noise above half the sample rate will appear as aliased noise and interfere with signals in the data system passband. A low-pass filter is usually used to control this aliased noise and the user is confronted with the problem of designing this filter.

To start, the sample rate of McVCO with the second channel operating is 330 samples/s so the attenuation of the filter at $165 \mathrm{~Hz}$ is of concern. In deciding what this attenuation should be several issues should be considered. First, does it matter? If the input has no frequency components above $165 \mathrm{~Hz}$ or a low resolution measurement is sufficient then no filtering may be required.

On the other hand, if sensitive measurements in the presence of noise are required then the filter is important. The maximum dynamic range of $\mathrm{McVCO}$ is limited by the 12 bit ADC to $72 \mathrm{~dB}$. If McVCO's output travels through radio and phone links then this range is further degraded and designing for a noise floor $60 \mathrm{~dB}$ below the maximum signal level is realistic. The noise floor contains all noise not just aliased noise so bandwidth limiting to the frequency range of interest is another factor to consider. Finally, if data collected through the telemetry network is subject to post processing then the phase response of the filter may need to be considered.

Selecting a filter response is often a complex compromise determined by the requirements of the application. Some filters have sharper roll offs, others have flatter passbands, and others have better phase responses. There is no perfect filter and the user must decide what characteristics are most important. 


\section{McVCO Test Cycle}

At reset or at 24 hour intervals, $\mathrm{McVCO}$ executes a test routine intended to give the user a qualitative idea of the instrument's condition. Units with ID numbers greater than 125 perform the tests every 18 hours. The following gives a description of the test cycle and suggests how it might be used. Some early versions may not have all the elements described. The version made for the Pacific Northwest Seismic Network (PNSN) has a different test cycle.

The test cycle is divided into three parts. To start, a $21.25 \mathrm{~Hz}$ tone modulates the subcarrier to signal the start of the cycle to the data system. This is followed by a series of eight seismometer test pulses. The cycle finishes with thirteen pulses representing the instrument gain setting and an instrument identification number. Later units with ID numbers more than 125 add twelve more pulses when they are in single channel mode. These pulses are the result of a second channel ADC measurement. Usually this ADC measurement is the site battery voltage. This use of the second channel is described in Part III, Monitoring Site Battery Voltage.

The total time of the test cycle depends on how it is started. If it was entered from a reset, then 10 seconds are required for autozero. This is followed by $10.25 \mathrm{~s}$ of the $21.25 \mathrm{~Hz}$ tone. Next the seismometer is tested for $18 \mathrm{~s}$ and the Gain-ID pulses take $13 \mathrm{~s}$. If the second channel ADC is used during the test cycle, $12 \mathrm{~s}$ more are needed.

During autozero, the McVCO output is set by the processor to the channel center frequency. Consequently it provides a zero reference level for discriminators and a field check for the correct channel frequency. Reference levels are also provided by the gain, ID, and second channel ADC pulses that are artificial subcarrier modulations at 0 and $\pm 62.5 \mathrm{~Hz}$ from center frequency. The $21.25 \mathrm{~Hz}$ tone modulates at $\pm 93.75 \mathrm{~Hz}$. Many discriminators have low-pass filters with $-3 \mathrm{~dB}$ corner frequencies at $20 \mathrm{~Hz}$. If this is the case, the $21.25 \mathrm{~Hz}$ amplitude will be the same as the gain, ID, and second channel ADC pulses at the discriminator output.

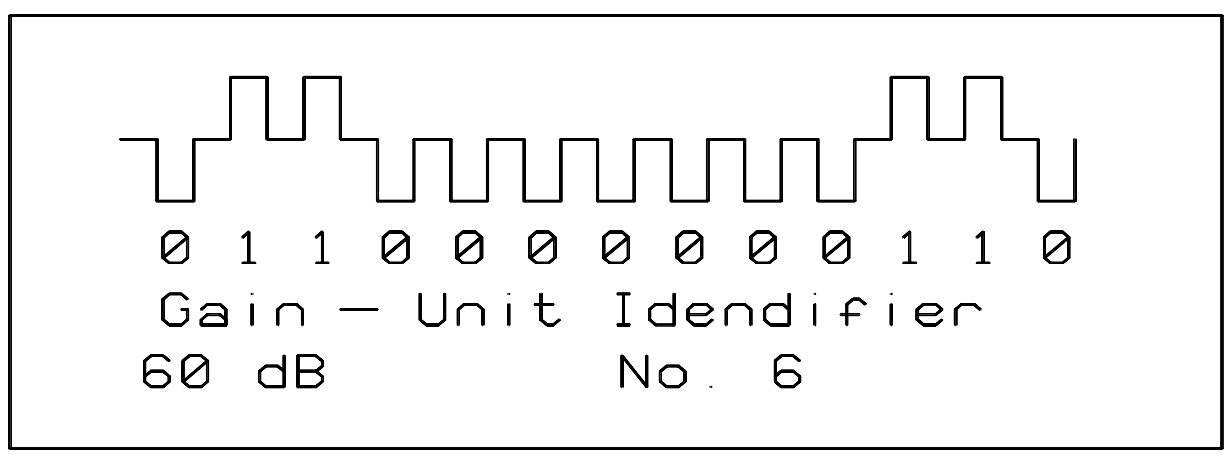

Figure 6. Demodulated gain and ID pulse train. 
Part III: Application Notes

In Figure 6, the first three pulses show the gain setting of the instrument's dip switch the last time the unit was reset. The last ten pulses are a binary number, msb first, which is the unit identifier. This number is programmed into the microcontroller. On their own they also show discriminator health. Each pulse is $0.5 \mathrm{~s}$ long and well within the discriminator bandwidth. The demodulated output should show sharply rising edges with around $20 \%$ overshoot. The tops and bottoms of waveforms should not sag.

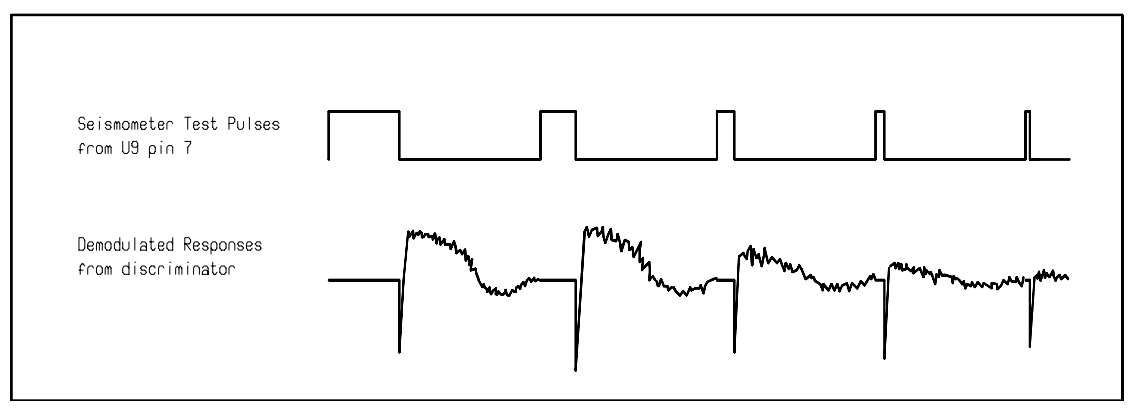

Figure 7. Seismometer test pulses and typical response.

The seismometer test pulses, shown in the upper trace of Figure 7, are signals from the processor injected into the input of the seismic-signal amplifier (see Figure 3A). Five of the eight pulses are shown in the figure. The first pulse is $1 \mathrm{~s}$ long followed $2 \mathrm{~s}$ later by the second pulse. Each pulse is half the length of the one before and follows it by $2 \mathrm{~s}$. The seismometer coil receives a current during each pulse and the seismometer mass moves a distance roughly proportional to the pulse length.

The lower trace in Figure 7, is the demodulated response of the instrument to the test pulses. While the first pulse is applied to the seismometer, McVCO is set to run at the channel center frequency. When the pulse ends, the instrument begins to make an ADC measurement and modulate the frequency output. The first thing the ADC sees is the recovery of the seismic-signal amplifier from the test pulse. This is shown on the lower trace as the sharp negative spike followed by the rise to the positive peak. The peak represents the damped response of the seismometer to the test pulse plus seismic signal and noise. After $2 \mathrm{~s}$ of ADC measurement, the instrument is set again at center frequency while the next pulse is applied to the seismometer. Measurement begins again with the ending of the test pulse and so on for a total of eight pulses.

The seismometer response to these test pulses depends on too many things to be exactly specified. Among other things it depends on seismometer type, $\mathrm{S}$ and $\mathrm{T}$ damping and attenuation, and the amplifier gain setting. In general the lower trace is a signature for the station that should be recorded and compared with previous records. The size of the response should not vary although superimposed seismic signal and noise may alter its appearance. Reductions in response may 
show problems with the seismometer such as tilt or moisture problems. An open cable or seismometer will show signs of the test pulse without the seismometer response, while an open $\mathrm{S}$ and $\mathrm{T}$ header will show neither the pulse nor the response.

The test routine is of most use to those who can view the demodulated output of McVCO but there are some features of the test cycle that can be observed directly. The autozero causes the unit to sit at the channel center frequency for $10 \mathrm{~s}$. Anytime this frequency needs to be checked it is only necessary to reset the unit while looking at the output with a frequency counter. The seismometer test pulse response cannot be seen without demodulation but the test pulse itself is useful for troubleshooting the seismic-signal amplifier. The test pulses inject a signal between 1 and $2 \mathrm{mV}$ at the amplifier input that can be followed with a scope to verify its operation. To generate the signal, reset the unit and $20 \mathrm{~s}$ later the pulses will appear. If a scanner or audio amplifier is available, the $21.25 \mathrm{~Hz}$ tone and the Gain-ID pulses can be heard. The Gain-ID pulses are slow enough and big enough that they can be interpreted by listening or viewing the modulated carrier with a scope. 


\section{Monitoring Site Battery Voltage}

McVCOs with ID numbers greater than 125 have the ability to use the second analog to digital converter (ADC) channel to measure battery voltage or other signals as part of the periodic test cycle. The interval of the test cycle in these later units was changed from 24 to 18 hours so that the time of measurement changed by six hours each day. The change in measurement time was made to assist in monitoring solar powered sites that have a daily cycle of charge and discharge. Other signals could be interfaced to use this ADC measurement but the site battery voltage is intended as the main application and is described here.

The second ADC channel is only available for use during the test cycle when the instrument is configured for single channel telemetry and the second ADC channel is not connected for low gain seismic signal measurement. See the previous note, Using the Second Channel, for additional information. To enable the second channel ADC during the test cycle, the jumpers at pins 15 and 16 of U9 are set for single channel operation with the test cycle on.

$$
\begin{aligned}
& \text { Pin } 15=5 \mathrm{~V} \text { (test cycle on }) \\
& \text { Pin } 16=0 \mathrm{~V}(\text { single channel telemetry })
\end{aligned}
$$

It is necessary to construct an interface circuit to bring the battery voltage within the 0 to 5 volt range of the ADC. The circuit can be a simple voltage divider. At the University of Alaska Geophysical Institute, the divider was built on a DIP header so that it could be installed in the field by plugging the header into the socket for U15. See Figure 3A. The header was wired as follows:

$316 \mathrm{~K} 1 \%$ resistor between pins 6 and 8

$34.8 \mathrm{~K} 1 \%$ resistor between pins 4 and 6

A wire from pin 8 to the $\mathrm{B}+$ solder point

Unbuffered dividers like this are loaded by the ADC but this may not matter as long as the scale factor is measured. The Geophysical Institute reports good linearity and a scale factor of 80 counts per volt.

If a buffered divider is required, the op amp at pins 1, 2 and 3 of U3 can be used as an inverting amplifier. This cancels the inversion at U15. For unity gain to the ADC, R48 should equal R25. A 5 to 1 divider brings the unloaded solar panel voltage of around 21 volts within the ADC span. Wire the divider between $\mathrm{B}+$ and ground. The second level shift pot (P4) can be used to adjust the calibration.

The ADC measurement pulses are the last 12 pulses of the test cycle. The last pulse is the least significant bit. Ones are positive pulses (positive deviations from center frequency). Zeros are negative pulses (negative deviations from center frequency). There is a rest period at center 
frequency between each pulse. See the figure in the previous note, McVCO Test Cycle, for a drawing of the 13 similar Gain and ID pulses. When the second channel ADC conversion is enabled there is a total of 25 of these pulses. The pulses can be checked by entering a test cycle after a reset.

Do not expect the battery voltage of a solar power system to remain nearly constant between measurements. If the battery is charging and discharging, internal losses add and subtract from the open circuit voltage of the battery. Changes of over a volt can occur when the battery goes from charge to discharge. When interpreting measurements remember to include any voltage drops from diodes between the battery and $\mathrm{McVCO} \mathrm{B}+$. 


\section{Using the Switched Power Output}

$\mathrm{McVCO}$ is designed to work in solar powered remote locations where access is difficult or impossible for parts of the year. The common problem of stations in these areas is intermittent power. Usually battery sulfation is to blame. If sulfation has occurred, power is available only when there is sun on the solar panel. The station drops out at night and comes on again in the morning. McVCO reacts to this situation by switching itself off when the power input voltage is too low and turning itself back on when it has risen to sufficient levels. See the circuit description in Part II for details. Switching off avoids dumping noise down the telemetry path when circuits are under powered, while switching on provides a clean reset for the microcontroller.

The radio transmitter and not $\mathrm{McVCO}$ is the biggest load on the power system. Some newer transmitters draw 80 to $130 \mathrm{~mA}$ depending on output power settings while McVCO uses around $20 \mathrm{~mA}$. Switching the transmitter off when McVCO is off, has the advantage of stopping further useless, possibly harmful battery discharge and decreasing the time the station is down. McVCO's switched power output, $\mathrm{SW}+$, is intended for this.

However there are limitations to using SW+ that should be considered before attaching a load to it. The method of sensing the battery condition by monitoring its voltage at McVCO's power input has weaknesses. First, the terminal voltage does not indicate the battery's state of charge and ability to supply power, particularly when the battery suffers from plate sulfation. Second, any resistance in the wiring between the battery and the instrument, including the battery's internal impedance, produces a voltage loss proportional to the current (IR loss) at McVCO's power input. This is usually not a problem because of the instrument's low current drain but if a load is powered through $\mathrm{SW}+$, its current increases the IR loss. This increased IR loss can lead to oscillation if switching on the SW+ load causes the voltage at McVCO's power input to fall to the point where the instrument turns itself off.

The oscillation sequence is as follows. McVCO is OFF while the battery voltage recovers. When the battery voltage reaches the ON threshold (11.5 V), McVCO turns ON and resets. McVCO turns the SW+ load ON. The increased current due to the load at SW+ drops the voltage at McVCO's power input below the critical level $(10.5 \mathrm{~V})$ and McVCO turns OFF and turns OFF $\mathrm{SW}+$. The voltage at the power input rises because there is no longer any IR loss or load on the battery. McVCO turns $\mathrm{ON}$ and resets etc.

When the main part of the voltage loss is due to line resistance, the oscillation can take place at a high frequency and could cause interference on the telemetry network. Two ways have been tried to control the frequency of oscillation. Early units had an RC delay built into the SW+ switch that kept the frequency of oscillation low. In later units, the microcontroller turns on SW+ after the $10 \mathrm{~s}$ autozero on reset. This approach stops any interference to the telemetry network because the instrument is set to the center channel frequency during autozero. The reset itself is so fast that the unit appears to be stuck at this frequency, while the transmitter turns on for a very short time. 
All this does not mean that SW+ should never be used. The load switch can be used successfully if certain precautions are taken. The difference between the ON and OFF switch points at McVCO's power input is one volt. If a safety margin of half a volt is allowed then with a total current load of $125 \mathrm{~mA}$ for both the transmitter and $\mathrm{McVCO}$, the total resistance of the battery and wiring must be kept to $4 \Omega,(0.5 \mathrm{~V} / 125 \mathrm{~mA})$. Wire size is not the problem unless there is a long run of wire between McVCO and the battery. The resistance of 22 gauge wire is $0.053 \Omega$ / meter. The problem is corrosion at battery terminals, wire connectors and terminal strips. Care should be taken to prevent corrosion by sealing these connection points with solder, grease or battery sealer.

Air cells (zinc-air and air alkaline cells) are some times used as backup power for solar power systems. They have fairly high internal resistances. When air cells reach the end of their service lives, the combination of reduced voltage and high resistance creates conditions favorable to $\mathrm{McVCO}$ power switch oscillation. Use of McVCO with systems that have air cell backup power systems is not recommended. The best place to put a power switch is at the battery and not at the VCO. Many newer solar power charge controllers have low voltage disconnects (LVD) which switch power at the battery and are less likely to oscillate than the McVCO power switch. 


\section{Three Component Connection}

McVCO boards may be daisy-chained through $\mathrm{J} 1$ and $\mathrm{J} 2$ modular connectors to permit three component observations of seismic events. Three McVCOs are chained with the J1 output of the first board connected to the $\mathrm{J} 2$ input of the second. The second board's J1 is connected to J2 of the third. The third board's output then has the subcarriers for all three components. If the boards operate in dual gain mode, then two audio subcarriers are produced and a three component site can produce as many as six subcarriers. Adjusting the amplitudes of each subcarrier is necessary so that they are equal at the output of the last McVCO board in the chain.

When McVCO boards are connected through $\mathrm{J} 1$ and $\mathrm{J} 2$, the subcarrier(s) from one board are summed with the subcarrier(s) of any previous boards. This subtotal is passed on and summed with the subcarrier(s) of the next until the last board in the chain has all the subcarriers present in its output. See Figure 8. The output circuit of each stage causes a loss of signal amplitude so that those subcarriers that pass through the most stages are attenuated the most. This uneven attenuation complicates equalization.

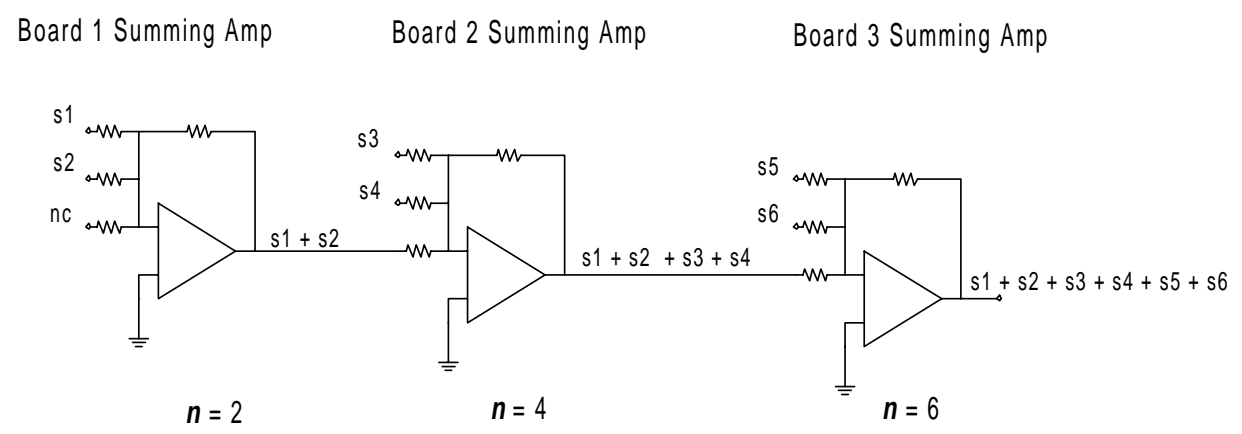

Figure 8. The output summing amps on each board combine the subcarriers of that board with the subcarriers from previous boards. When $s 1=s 2=\ldots s n$, the rms value of the output from each board is proportional to the square root of the number of subcarriers, $n^{1 / 2}$.

Equalization can be checked with a spectrum analyzer or a personal computer equipped with spectrum analysis hardware and software but these instruments may not be available. The method that follows requires a true rms voltmeter to measure subcarrier levels when adjustments are made. The procedure can be done in the field.

Complex waveforms composed of sinewaves of equal amplitude, have a simple relationship between the total rms voltage and the rms voltage that is common to each part. Where $\boldsymbol{n}$ is the number of parts and $\boldsymbol{V}_{\mathrm{S}}$ is the common rms voltage of each part; the total rms voltage $\left(\boldsymbol{V}_{\mathrm{TOT}}\right)$ of the complex waveform is,

$$
\boldsymbol{V}_{\mathrm{TOT}}=\left(\boldsymbol{V}_{\mathrm{S}}\right)\left(\boldsymbol{n}^{1 / 2}\right)
$$


To use this relationship for equalizing multiple component sites, start with the desired output, $\boldsymbol{V}_{\text {Тот }}$. If $\boldsymbol{V}_{\text {Tот }}$ is unknown, obtain it from the specifications for the device, such as a radio transmitter, which accepts it as input. If the specifications are not available, guess. $\boldsymbol{V}_{\text {Tот }}$ can be readjusted at the end of the procedure after the components are equalized. A good trial value of $\boldsymbol{V}_{\text {тот }}$ is $225 \mathrm{mV} \mathrm{rms.}$

To equalize the subcarriers in the output of chained McVCO boards, complete the following adjustments in the described order.

1. Where $\boldsymbol{n}$ is the total number of subcarriers in the output of the last board in the chain, adjust each subcarrier on every board to $\boldsymbol{V}_{\mathrm{S}}$, for $\boldsymbol{V}_{\mathrm{S}}=\boldsymbol{V}_{\mathrm{TOT}} /\left(\boldsymbol{n}^{1 / 2}\right)$. Measure the component at TP5 and adjust P5. If the board is operating in dual gain mode, measure the second subcarrier at TP6 and adjust $\mathrm{P} 6$.

2. Starting at the first board in the series, measure its rms audio signal level between CAR+ and CAR- test points on the left edge of the board. Adjust P7 so that the board output is $\left(\boldsymbol{V}_{\mathrm{S}}\right)\left(\boldsymbol{n}^{1 / 2}\right)$, where $n$ is the number of all subcarriers in the output of that board. Go to CAR+ and CAR- of the next board in the chain and adjust P7 on that board for the new value of $\boldsymbol{n}$ as required by the number of subcarriers in the output of this board. See Figure 8 for an example of how $\boldsymbol{n}$ changes. Continue until the last board.

3. P7 on the last board adjusts level of the total equalized waveform. Measure between CAR+ and CAR- while adjusting for the known value of $\boldsymbol{V}_{\text {Tот }}$ or monitor the response of the device which uses the summed subcarriers as its input. If the initial value of $\boldsymbol{V}_{\mathrm{S}}$ is wrong and the required signal level is outside the adjustment range of $\mathrm{P} 7$ on the last board change $\boldsymbol{V}_{\mathrm{S}}$ and repeat the procedure.

For example, high and low gain observation of a three component seismic site is required. Three $\mathrm{McVCO}$ boards which each generate two subcarriers are connected through $\mathrm{J} 1$ and $\mathrm{J} 2$ for a total of six subcarriers. Figure 8 shows how the subcarriers combine. The telemetry is via a low power radio transmitter. The desired deviation of the transmitter is $\pm 2.5 \mathrm{KHz}$. To adjust the amplitudes of the subcarriers, the rms value of the modulation which produces this deviation must be found.

The deviation sensitivity of the transmitter is set by the manufacturer for $5 \mathrm{KHz}$ deviation at $1.0 \mathrm{~V}$ rms modulation. Deviation is proportional to the peak voltage and not the rms voltage. The manufacturer uses a single tone to specify modulation characteristics, consequently the peak deviation is $5 \mathrm{KHz} /\left(2^{1 / 2}\right)(1.0 \mathrm{~V} \mathrm{rms})=3.54 \mathrm{KHz} / \mathrm{V}$. Using the peak deviation sensitivity, the peak modulating voltage for $2.5 \mathrm{KHz}$ deviation is $(2.5 / 3.54) \mathrm{V}$ peak $=0.71 \mathrm{~V}$ peak.

To equalize the subcarriers, the rms value of the modulating voltage is needed. Unfortunately, there is no simple way to convert a complex waveform from peak to rms values. However, an approximation can be made. Table 1 shows the mean crest factor for equalized waveforms composed of telemetry frequencies $(680,1020, \ldots 3060)$. The crest factor is the ratio of peak 
voltage to rms voltage. From Table 1, a six component waveform has a mean crest factor of 2.21. Therefore, the approximate rms value of the modulating voltage is $0.71 \mathrm{~V}$ peak $/ 2.21=321 \mathrm{mV}$ rms.

\begin{tabular}{|c|c|c|c|c|c|c|c|c|}
\hline $\begin{array}{c}\text { Number of } \\
\text { components }\end{array}$ & 1 & 2 & 3 & 4 & 5 & 6 & 7 & 8 \\
\hline Mean Crest Factor & $2^{1 / 2}$ & 1.92 & 2.12 & 2.18 & 2.20 & 2.21 & 2.22 & 2.23 \\
\hline
\end{tabular}

Table 1. The mean Crest Factor of complex telemetry waveforms with different numbers of components. The components have equal amplitudes and their frequencies are the seismic telemetry frequencies $(680,1020, . .3060)$. Crest Factor is the ratio, V peak / V rms. The calculation of Crest Factor was made by summing all possible combinations of telemetry frequencies at random phases for many trials to find the mean peak to peak voltage for each waveform with more than one component.

The value of each subcarrier $\left(\boldsymbol{V}_{\mathrm{S}}\right)$ is then $\boldsymbol{V}_{\mathrm{S}}=(321) /\left(6^{1 / 2}\right)=131 \mathrm{mV} \mathrm{rms}$. Step 1 of the procedure is completed by setting each subcarrier on every board to $131 \mathrm{mV}$ rms at TP5 or TP6 on the respective board with P5 and P6.

Board 1 has two subcarriers in its output. P7 on Board 1 should be adjusted so that between the board's CAR+ and CAR- test points the output is $131\left(2^{1 / 2}\right) \mathrm{mV} \mathrm{rms}=185 \mathrm{mV} \mathrm{rms}$.

Board 2 has four subcarriers in its output. $\mathrm{P} 7$ on Board 2 should be adjusted so that between the board's CAR+ and CAR- test points the output is $131\left(4^{1 / 2}\right) \mathrm{mV} \mathrm{rms}=262 \mathrm{mV} \mathrm{rms}$.

Board 3 has six subcarriers in its output. P7 on Board 3 should be adjusted so that between the board's CAR+ and CAR- test points the output is $131\left(6^{1 / 2}\right) \mathrm{mV}=321 \mathrm{mV} \mathrm{rms}$.

This completes the example adjustment. The equalized 321 millivolt rms waveform will produce an average of $\pm 2.5 \mathrm{KHz}$ transmitter deviation. 